hep-th/0512102

PUPT-2184

\title{
Random Matrices and the Spectrum of N-flation
}

\author{
Richard Easther ${ }^{a} 1$ and Liam McAllister ${ }^{\text {国 }}$ \\ ${ }^{a}$ Department of Physics, Yale University, New Haven CT 06520 \\ ${ }^{b}$ Department of Physics, Princeton University, Princeton NJ 08540
}

\begin{abstract}
$\mathrm{N}$-flation is a promising embedding of inflation in string theory in which many string axions combine to drive inflation [1]. We characterize the dynamics of a general N-flation model with non-degenerate axion masses. Although the precise mass of a single axion depends on compactification details in a complicated way, the distribution of masses can be computed with very limited knowledge of microscopics: the shape of the mass distribution is an emergent property. We use random matrix theory to show that a typical N-flation model has a spectrum of masses distributed according to the Marčenko-Pastur law. This distribution depends on a single parameter, the number of axions divided by the dimension of the moduli space. We use this result to describe the inflationary dynamics and phenomenology of a general N-flation model. We produce an ensemble of models and use numerical integration to track the axions' evolution and the resulting scalar power spectrum. For realistic initial conditions, the power spectrum is considerably more red than in single-field $m^{2} \phi^{2}$ inflation. We conclude that random matrix models of N-flation are surprisingly tractable and have a rich phenomenology that differs in testable ways from that of single-field $m^{2} \phi^{2}$ inflation.
\end{abstract}

December 2005

\footnotetext{
1 richard.easther@yale.edu

2 lmcallis@princeton.edu
} 


\section{Contents}

1. Introduction . . . . . . . . . . . . . . . . . . . . . . . . . . . . . . . 1

2. The N-flation Model . . . . . . . . . . . . . . . . . . . . . . . . . 5

2.1. Basic Form of the Model . . . . . . . . . . . . . . . . . . . . . . . . . . 5

2.2. N-flation in a Stabilized Vacuum . . . . . . . . . . . . . . . . . . . . . . 6

2.3. F-flatness Conditions . . . . . . . . . . . . . . . . . . . . . . . . 8

3. Mass Terms in N-flation . . . . . . . . . . . . . . . . . . . . . . . . . . . 9

3.1. The Quadratic Potential . . . . . . . . . . . . . . . . . . . . . . . 10

3.2. The Complete Potential . . . . . . . . . . . . . . . . . . . . . . . . . . . 12

4. Random Matrix Treatment of the Mass Terms . . . . . . . . . . . . . . . . . . . 13

4.1. Statistical Model for the Covariant Derivatives . . . . . . . . . . . . . . . . . 14

4.2. Axion Masses and the Marčenko-Pastur Law . . . . . . . . . . . . . . . . . . 17

4.3. Some Relations to Other Results in Random Matrix Theory . . . . . . . . . . . 19

5. Monte Carlo Analysis of Random Mass Matrices . . . . . . . . . . . . . . . . . . 20

5.1. Properties of the Component Matrices . . . . . . . . . . . . . . . . . . . . 20

5.2. Results of Monte Carlo Simulation . . . . . . . . . . . . . . . . . . . . . . 21

6. N-flation Dynamics and Initial Conditions . . . . . . . . . . . . . . . . . . . . 22

6.1. Inflationary Dynamics . . . . . . . . . . . . . . . . . . . . . . . . . . . 22

6.2. Initial Conditions . . . . . . . . . . . . . . . . . . . . . . . . . . . . . 25

6.3. Number of E-folds . . . . . . . . . . . . . . . . . . . . . . . . . . . . . 27

7. Generation of Adiabatic Perturbations . . . . . . . . . . . . . . . . . . . . . . 31

8. Astrophysical Constraints on the N-flation Parameters . . . . . . . . . . . . . . . 36

9. Possible Exceptions to our Results . . . . . . . . . . . . . . . . . . . . . . . . 39

9.1. Conditions for a Trans-Planckian Displacement . . . . . . . . . . . . . . . . 40

9.2. Conditions for an N-flation Model with Marčenko-Pastur Spectrum . . . . . . . . 42

10. Conclusions . . . . . . . . . . . . . . . . . . . . . . . . . . . . . . . . . 44

Appendix A. Statistical Suppression of Cross-Couplings . . . . . . . . . . . . . . . . 47

Appendix B. Inflation in the Presence of Cross-Couplings . . . . . . . . . . . . . . . 48

\section{Introduction}

Inflationary model-building in string theory [2,3] is advancing rapidly, impelled by the strong and growing evidence for inflation, and facilitated by a series of technical advances [4,50,6] in string theory. Among the inflationary models that have remained difficult to embed in string theory are those known as large-field models, in which the inflaton vacuum expectation value (vev) varies by much more than one Planck mass during inflation [7]. This class includes what are arguably the simplest inflationary potentials, those quadratic [8] or quartic in the inflaton.

The controllability of these models is a subject of debate [9], and depends strongly on the relation between particle physics and gravity near the Planck scale. Many authors 
take the view that configurations with trans-Planckian vevs are controllable provided that the energy density is well below the Planck scale. Because large-field inflation requires a very weakly coupled field, potentials such as $\lambda \phi^{4}$ and $m^{2} \phi^{2}$ automatically have safely sub-Planckian energy densities. This argument is reliable if we restrict our attention to quantum corrections that involve higher-order terms in the spacetime curvature $\mathcal{R}$, since $\mathcal{R} \ll M_{p}^{2}$ during the portion of inflation that is observationally relevant. In a model that does not unify gravity with the other forces we might ignore gravitationally induced corrections to the particle physics effective potential, and only worry about quantum corrections to the spacetime background. In this context, trans-Planckian vevs can indeed be benign.

However, gravitationally-suppressed couplings between the fields that contribute to $T_{\mu \nu}$ generically appear in models that unify gravity and particle physics, such as supergravity and superstring models. The problem is that loop corrections generically become large when vevs, instead of energy densities, exceed the Planck scale; for example, it is very difficult to forbid corrections to the Kähler potential that are controlled by field vevs in Planck units. Experience with supergravity and superstring models strongly suggests that Planck scale vevs usually imply large corrections to the inflaton effective potential. This observation has been used to put bounds on inflationary observables, such as the tensor power spectrum [10].3

Our view is that because trans-Planckian vevs are always dangerous, if not necessarily fatal, in string theory and supergravity models, it is worthwhile to construct specific models in which these problems can be overcome. In the best case, understanding transPlanckian vevs in a concrete string theory construction would give a useful toy model for understanding general quantum gravity corrections to large-field models.

Dimopoulos, Kachru, McGreevy, and Wacker [1] have proposed a clever solution to this set of problems. 1 Combining $N \gg 1$ fields with sub-Planckian displacements into a single effective inflaton, they obtained $m^{2} \phi^{2}$ inflation without having to build a trans-Planckian single-field displacement. The resulting model, which they called N-flation, is an example of assisted [15, 13,16, 17, 18] inflation. They further explained that N-flation can be embedded in string theory by choosing the $N$ fields to be axions. Axions are typically present in large numbers in string compactifications, and even when all other moduli are stabilized,

3 There are a few exceptions to this rule [11, 12].

4 For earlier work in this direction, see 13]. For an application of these ideas to quintessence, see [14]. 
the axion potentials remain rather flat as a consequence of well-known nonrenormalization theorems [19].

The axions are pseudo-Nambu-Goldstone bosons associated with nonperturbativelybroken shift symmetries. Hence, N-flation may be categorized as an approximately shiftsymmetric, closed string, large-field model. been suggested [3] as a natural origin of flat inflaton potentials in string theory; however, the effects that break such open string shift symmetries [22] are often too strong to leave a suitably flat potential. (See also [23] for a general explanation of the role of approximate shift symmetries in preserving flat potentials.)

When the individual axion displacements are small in Planck units, the leading term in the potential is a mass matrix that connects the $N$ axion fields. Choosing a basis in which the kinetic terms are canonical and the mass terms are diagonal, the data of an N-flation model becomes simply a list of $N$ masses. The authors of [1] focused their attention on the special case in which the masses are identical, and showed that in that case the observational predictions are precisely those of $m^{2} \phi^{2}$ chaotic inflation. They argued that inflation still occurs in the general case with non-degenerate masses, with qualitatively similar results. The dynamics is somewhat more complicated, however, as the most massive axions relax first to their minima, diminishing the Hubble friction and causing the lighter axions to roll more rapidly.

The purpose of this note is to give a quantitative treatment of this general case of N-flation, in which all the masses are distinct. Two natural questions arise in this context. First, what are the dynamics and the observational predictions of an N-flation model with a given set of masses $m_{i}$ ? In particular, how does a spread in masses affect the spectral index $n_{s}$ ? Second, what spectrum of masses should one expect from microscopic considerations? The mass of each axion depends on a formidable array of compactification details. In the context of KKLT moduli stabilization [5], these details include some as-yet uncomputed factors, such as fluctuation determinants for Euclidean D3-branes, in addition to betterknown but highly unwieldy expressions involving the fluxes and the Kähler potential. Computing the precise masses of $N$ axions in a nontrivial, stabilized compactification would therefore appear to be extremely difficult. Might we nevertheless be able to deduce something about the typical spacing of axion masses?

5 Natural inflation [20,21, in contrast, is a small-field model driven by a single axion. 
Surprisingly, we find an essentially universal result for the axion masses in N-flation: the probability distribution for the masses-squared is given by an analytic formula known as the Marčenko-Pastur law. The shape of this curve depends on a single parameter $\beta$ determined by the dimensions of the Kähler and complex structure moduli spaces. We describe this result as universal because it does not depend on specific details of the compactification, such as the intersection numbers, the choice of fluxes, or the location in moduli space. The result is also insensitive to superpotential corrections such as threshold corrections and instanton determinants. The shape of the mass distribution depends only on the basic structure of the mass matrix, which is specified by the supergravity potential. (The overall mass scale is not determined a priori; as in [1], we fix it by requiring the observed amplitude of density perturbations.)

This welcome situation arises because of the size (typically larger than $200 \times 200$ ) of the matrices involved: the distribution of the eigenvalues of random matrices is most easily characterized when the matrices are large. In this way, the simplifications enjoyed by large random matrices [24,25,26,27] help N-flation to generate a prediction for the scalar spectral index which is substantially (though not completely) independent of compactification details. This is a striking example of a problem that is tractable because of, rather than in spite of, the high dimensionality of the scalar field space in string theory.

Equipped with the Marčenko-Pastur law for the mass distribution, we numerically integrate the equations of motion for a large sample of realizations of N-flation. We find that for a range of initial conditions and for most values of $\beta$, the scalar power spectrum is typically more red (i.e. farther from scale-invariant) than in the case where all the masses are identical.

In addition to developing a random matrix theory calculation of the axion mass spectrum, we treat the inflationary dynamics and perturbation spectrum produced by N-flation. (For related earlier work, see [28,29]. Additionally, Kaloper and Liddle [17] have considered inflation driven by a tower of Kaluza-Klein fields with quadratic potentials. However, this mass distribution is very different from the Marčenko-Pastur form relevant in our case of string axions.) It turns out that while there are very general results for the perturbation spectrum produced by multi-field inflation (e.g. [30]), the spectrum of N-flation is surprisingly complex and has several previously unsuspected features. In this paper, we work only with the slow-roll approximation, and the results here represent an initial survey rather than a comprehensive analysis of the subject. We would like to emphasize that the dynamics and the resulting inflationary power spectrum do not become independent of the 
initial conditions at late times [17]. Thus, even if one had a microscopically computable N-flation model, some of the predictions would depend on the initial conditions. For this reason, we present our results as functions of the initial conditions, illustrating the simplifications that arise in a few special cases. A theory of the initial conditions, which we do not address in this paper, would still be necessary for a complete understanding of this class of models.

Readers whose main interests are the inflationary dynamics and the predictions of the model, rather than the problem of the axion mass spectrum in a string compactification, could begin with $\S 2.1$, examine the mass spectrum of $\S 4.2$, and then proceed from $\S 6$.

\section{The N-flation Model}

\subsection{Basic Form of the Model}

Here we briefly recall the structure of the model proposed by Dimopoulos et al.[1]. Consider $N$ axions $\varphi_{i}$ whose periodic potentials

$$
V\left(\varphi_{i}\right)=\Lambda_{i}^{4}\left(1-\cos \left(\frac{\varphi_{i}}{f_{i}}\right)\right)
$$

are independent and arise solely from nonperturbative effects. As we will explain, we omit multi-instanton corrections to this potential. The scale $\Lambda_{i}$ is determined by dimensional transmutation and can be hierarchically small compared to the ultraviolet cutoff. We find it convenient to absorb the axion decay constants $f_{i}$ via

$$
\phi_{i} \equiv \frac{\varphi_{i}}{f_{i}}
$$

and we take units where $M_{p}=1$. For small axion displacements $\phi_{i} \ll 1$, the potential may be Taylor expanded, and the Lagrangian is 6

$$
L=\frac{1}{2} \nabla_{\mu} \phi_{i} \nabla^{\mu} \phi_{i}-\frac{1}{2} m_{i}^{2} \phi_{i}^{2}
$$

If the masses $m_{i}$ are very nearly equal and the axions are initially displaced by a common amount $\bar{\phi}$, they will roll in unison toward their joint origin. For all practical purposes, this is equivalent to the motion of a single field $\Phi$ with a displacement $\sqrt{N} \bar{\phi}$. It

6 For the moment we have neglected the important possibility of nontrivial axion kinetic terms, but we will come to a complete treatment of this point. 
follows that even if each axion is displaced by a distance in field space small compared to the Planck mass, $\Phi$ can satisfy the conditions for slow-roll inflation. In this sense, N-flation realizes $m^{2} \phi^{2}$ inflation in a very well-controlled string theory setting.

One should ask how often this simple potential involving $N$ decoupled axions with canonical kinetic terms and identical masses can arise in a string compactification. We would expect that a generic model with $N$ decoupled axions will have a nontrivial spectrum of masses. Our goal is to determine exactly which sorts of mass spectra are possible in string compactifications. Surprisingly, we will find that a single characteristic spectrum emerges, regardless of microscopic details.

\subsection{N-flation in a Stabilized Vacuum}

An important requirement for a realistic inflationary model in string theory is the stabilization of compactification moduli. For this reason, it is important to verify in detail that the particular classes of axion potentials needed for N-flation do arise in a class of stabilized string compactifications.

The moduli are typically paired with axions in complex combinations. In the type IIB theory, for example, the Kähler modulus $\rho_{i}$ measuring the volume of a given four-cycle $B_{4}^{(i)}$ combines with the axion

$$
\phi_{i}=\int_{B_{4}^{(i)}} C_{4}
$$

as $\rho_{i}-i \phi_{i}$. (We have chosen a convenient sign.)

There is a simple relationship between the moduli-stabilizing potentials and the axion potentials in the resulting stabilized vacuum. For definiteness, we will focus on moduli stabilization in type IIB string theory by the method of KKLT [5], in which all moduli are stabilized by the combination of fluxes [4] and a nonperturbative superpotential. In the type IIA vacua of [31], most fields receive masses from fluxes, but the lightest axions are lifted by nonperturbative effects; for this reason, we expect rather similar qualitative features in this context. $\mathrm{G}$ We leave the properties of N-flation in more general stabilized vacua as an interesting subject for future work.

The KKLT superpotential is

$$
W=W_{0}\left(S, \chi_{a}\right)+\sum_{i} A_{i}\left(\chi_{a}\right) e^{-a_{i} \tau_{i}}
$$

7 This conclusion was reached in discussions with A. Krause and A. Mazumdar. 
Here

$$
W_{0}=\int G_{3} \wedge \Omega
$$

is the flux-induced superpotential, which depends on the dilaton $S$ and on the $h_{2,1}$ complex structure moduli $\chi_{a}$. The sum of nonperturbative superpotential terms runs over all the Kähler moduli $\tau_{i} \equiv \rho_{i}-i \phi_{i} \quad\left(i=1 \ldots h_{1,1}\right)$. The real parts $\rho_{i}$ measure the volumes of four-cycles in the Calabi-Yau, and the imaginary parts $\phi_{i}$ are the axions arising from the four-form potential $C_{4}$. We will work at volumes large enough to ensure that neglecting the multi-instanton corrections to (2.5) is consistent (see $\S 9.1$ ).

We are assuming that there is one nonperturbative effect for each independent fourcycle. For each such cycle, there are two possible sources of a nonperturbative superpotential: Euclidean D3-branes or strong infrared dynamics in a gauge theory, for which gaugino condensation on a stack of $N>1 \mathrm{D} 7$-branes is the simplest example. The constants $a_{i}$ are either $2 \pi$, in the case of Euclidean D3-branes, or $2 \pi / N$ for gaugino condensation. For simplicity we will assume that Euclidean D3-branes provide all the nonperturbative superpotential terms; relaxing this assumption is straightforward and does not affect our results. The prefactors $A_{i}\left(\chi_{a}\right)$, although knowable in principle, are unknown in practice. They stem from determinants of fluctuations on the D3-brane-instanton worldvolume.

Finally, the Kähler potential is given, to leading order, by

$$
K=-2 \log (\mathcal{V})
$$

where $\mathcal{V}$ is the Calabi-Yau volume, amounting to a highly nontrivial combination of the various Kähler moduli $\rho_{i}$. (When there is only a single Kähler modulus, $\mathcal{V} \propto \rho^{3 / 2}$.)

The only feature of the KKLT scenario that will be important for our discussion is that the superpotential is a sum of an axion-independent term [32] and $N$ nonperturbative terms in which the axions appear as phases. This would still be true in an appropriate generalization of the KKLT method to other string theories. When this is the case, the axions potentials arise exclusively from the same nonperturbative effect that stabilizes the Kähler moduli with which they are paired. Our qualitative conclusions do not even depend on this basic property, but for the purpose of presenting a definite computation, we do assume the KKLT form except where noted. 


\subsection{F-flatness Conditions}

We are interested in a scenario in which the axions' displacement from their joint minimum is the dominant source of positive energy during inflation. Once N-flation ends and the axions have relaxed to this minimum, supersymmetry may still be broken by some additional effect. However, this effect should be subleading, and ideally constant, during inflation, lest it ruin the useful properties of the axion-induced inflationary potential.

The F-terms $D_{A} W$ of the various moduli $S, \chi_{a}, \rho_{i}$ depend on the superpotential, and through it, on each of the axions. As the axions vary, the F-terms change, so the inflationary trajectory cannot be F-flat. However, we will substantially simplify our analysis by approximating the final vacuum as F-flat. This is an excellent approximation for phenomenologically viable models with low-energy supersymmetry: the F-term energy at late times can be at most of order the intermediate scale, and hence will be highly suppressed relative to the inflationary energy of the axions 8 Correspondingly, we will refer to configurations as F-flat even if they satisfy only the weaker condition that the F-term energy is very small compared to the energy scales of interest for inflation. We could also imagine that the dominant supersymmetry-breaking in the final state is accomplished by a D-term effect such as an anti-D3-brane. In any event, we require that as the axions roll to their minima, the configuration relaxes to a KKLT vacuum,

$$
V\left(\phi_{f}, S, \chi, \rho\right)=V_{K K L T}(S, \chi, \rho)
$$

with $\phi_{f}$ the value the axions take at their minima. To summarize, then, our picture is that the axions are displaced from the minima they take in a supersymmetric $A d S_{4}$ vacuum, and their potentials are unaffected by any additional, subleading supersymmetry-breaking effects.

We assume that all other moduli are fixed during inflation. By construction, all the moduli have nonzero masses in the KKLT vacuum, but we are requiring in addition that these masses are somewhat larger than the axion masses. A condition of this nature is essential to prevent unfixed moduli, whose potentials are not protected against corrections, from spoiling the properties of an inflationary epoch in which only the axions are dynamical. At first sight it may appear implausible that a modulus field $\rho_{i}$ would be firmly stabilized while its axion partner $\phi_{i}$ from the same chiral multiplet remains somewhat mobile. The

8 We thank S. Kachru for helpful discussions of this point. 
important difference is that the $\rho_{i}$ appear in the Kähler potential, whereas the $\phi_{i}$ do not; as a result, when the KKLT potential is expressed in terms of canonically-normalized fields, it is steeper in the $\rho_{i}$ directions than in the $\phi_{i}$ directions. Equivalently, the difference is that the $\phi_{i}$ enjoy the protection of an approximate shift symmetry, but the $\rho_{i}$, like the other moduli, do not. During inflation the unprotected moduli will typically acquire masses $m \gtrsim H$, while the axion masses will remain substantially smaller.9

To find the supersymmetric $A d S_{4}$ vacuum, we solve

$$
0=D_{A} W \equiv \partial_{A} W+\left(\partial_{A} K\right) W
$$

where $A$ runs over the dilaton $S$, the complex structure moduli $\chi_{a}$, and the Kähler moduli $\rho_{i}$. The F-terms (2.9) are axion-dependent, and cannot vanish unless $\phi=0$ or $\pi$. However, the F-terms also depend on the other moduli $S, \chi, \rho$. As explained above, while the axions are supporting inflation, the other moduli must remain fixed. Together with (2.8), this implies that the full axion potential is

$$
V(\phi)=V\left(\phi, \rho_{i}^{(0)}, \chi_{i}^{(0)}, S^{(0)}\right)
$$

i.e. the supergravity potential evaluated on a background configuration in which $\rho_{i}, \chi_{a}, S$ take the same values they take at the global minimum. Although the F-terms do not vanish along the axion trajectory, but only at the endpoints, one must still enforce

$$
D_{A} W\left(\phi_{i}=0\right)=0
$$

This amounts to setting the axions to zero in each of the F-terms and using the residual relations to constrain $\rho_{i}, \chi_{a}, S$. These constraints will provide very useful clues about the structure of the axion potential.

\section{Mass Terms in N-flation}

We will now work out in some detail the potential arising from a KKLT realization of N-flation.

9 See e.g. [33] for a discussion of related issues. 


\subsection{The Quadratic Potential}

Recall that the axion kinetic terms are identical in structure to those of the Kähler moduli with which they are paired, so that

$$
\mathcal{L}_{\text {axion }}=\frac{1}{2} M_{p}^{2} K_{i j} \nabla_{\mu} \phi^{i} \nabla^{\mu} \phi^{j}-V
$$

The supergravity potential is

$$
V=\exp \left(\frac{K}{M_{p}^{2}}\right)\left(K^{A B} D_{A} W D_{\bar{B}} \bar{W}-3 \frac{|W|^{2}}{M_{p}^{2}}\right)
$$

where, as before, $A, B$ run over the dilaton $S$, the complex structure moduli $\chi_{a}$, and the Kähler moduli $\rho_{i}$. (In this section we find it convenient to retain explicit factors of $M_{p}$, which we have set to unity elsewhere.)

In the KKLT superpotential (2.5), each nonperturbative factor depends on the associated axion as

$$
W_{i}=A_{i} e^{-2 \pi \rho_{i}} e^{2 \pi i \phi_{i}} \equiv C_{i} e^{2 \pi i \phi_{i}}
$$

We have chosen the notation $C_{i}$ to stress the fact that the moduli $\chi_{a}, \rho_{i}$ which appear in $C_{i}$ are not dynamical variables in the scenario of interest: by (2.9), these fields are fixed to the values they take in the supersymmetric minimum. Thus, the $C_{i}$ are constants as far as the inflationary dynamics is concerned. Because the axion $\phi_{i}$ appears only in $W_{i}$, and neither in the Kähler potential nor elsewhere in the superpotential, every factor of $e^{2 \pi i \phi_{i}}$ in (3.2) will appear multiplied by the constant $C_{i}$.

Inserting (2.5) in (3.2), performing a Taylor expansion around the origin $\phi_{i}=0$, and using the F-flatness conditions $\left.D_{A} W\right|_{\phi_{i}=0}=0$, we arrive at

$$
V=(2 \pi)^{2} \hat{M}_{i j} \phi^{i} \phi^{j}+\mathcal{O}\left(\phi^{3}\right)
$$

where

$$
\hat{M}_{i j}=\frac{1}{M_{p}^{2}} e^{K}\left(K^{A B} D_{A} C_{i} D_{B} C_{j}-3 C_{i} C_{j}\right)
$$

The kinetic terms (3.1) and the mass matrix (3.4) are evidently not diagonal in general. Thus, we find that the potential does not automatically take on the uncoupled form required for N-flation. That is, the mass matrix is typically not diagonal in the the basis in which the superpotential is simple, with a single axion appearing in each nonperturbative 
term.19 Moreover, the off-diagonal terms appear to be of the same order as the diagonal ones. We show in Appendix A that for essentially statistical (rather than directly physical) reasons, the cross-couplings in $\hat{M}_{i j}$ are actually suppressed relative to the diagonal terms. Even so, keeping track of these cross-couplings will provide a key insight into the mass spectrum.

At this stage, it is convenient to make a change of basis that renders the axion kinetic terms canonical. Define $O$ to be the matrix that diagonalizes the metric $K_{i j}$ on the Kähler moduli space,

$$
O_{i}{ }^{k} K_{k l} O_{j}^{l}=\kappa_{i}^{2} \delta_{i j}
$$

with $\kappa_{i}^{2}$ the positive eigenvalues. The eigenvalues $\kappa_{i}$ are related to the axion decay constants 11 through

$$
\kappa_{i}=\frac{f_{i}}{M_{p}}
$$

Next, rescale the $\phi_{i}$ to absorb the factors $\kappa_{i}$, so that the kinetic terms are canonically normalized and $K^{i j}$ is the $N \times N$ identity matrix. Finally, redefine the complex structure moduli and the dilaton so that $K^{a b}, a, b=1, \ldots P$ is the $P \times P$ identity matrix, and return to units in which $M_{p}=1$.

This leads to the Lagrangian density

$$
\mathcal{L}=\frac{1}{2} \partial_{\mu} \phi_{i} \partial^{\mu} \phi^{i}-M_{i j} \phi^{i} \phi^{j}
$$

where

$$
M_{i j}=(2 \pi)^{2} \frac{e^{K}}{f_{i} f_{j}} O_{i}{ }^{k}\left(D_{A} C_{k} D^{A} C_{l}-3 C_{k} C_{l}\right) O_{j}{ }^{l}
$$

Provided that the potential takes this purely quadratic form, i.e. neglecting the higher-order terms omitted in (3.4), there exists a further orthogonal rotation that diagonalizes $M_{i j}$. The result is that all the data specifying an N-flation model can be stored in the list of eigenvalues of (3.9), which are the masses-squared $m_{i}^{2}$ of $N$ canonicallynormalized, uncoupled axions. Our goal in the remainder of the paper is to characterize the statistical properties of these eigenvalues, based on the structure of (3.9).

10 We thank S. Kachru and J. Wacker for useful discussions of this point.

11 According to [34], the $\kappa_{i}$ are at most of order unity; see also [35]. 


\subsection{The Complete Potential}

In the previous section we focused on the quadratic potential (3.4). However, terms of higher order in the axions must be retained when some of the initial dimensionless axion displacements $\phi_{i}^{(0)}$ are of order one. When the quadratic approximation to the axion potential is invalid, it is generally impossible to diagonalize the potential to remove cross-coupling terms.

Because large initial displacements are advantageous for inflation, there is a competition between obtaining enough inflation and having a controllable expansion of the full potential (3.2) as a potential for uncoupled fields. For that reason, in this section we will present the full, unexpanded potential (3.2) and sketch the resulting axion dynamics.

To do this, we reorganize (3.2) and use the F-flatness conditions (2.11). The result from the F-term piece 12 of the potential is

$$
V=\sum_{i, j}\left(e^{K} K^{A B} D_{A} C_{i} D_{B} C_{j}\right)\left(1+\cos \left(2 \pi \phi_{i}-2 \pi \phi_{j}\right)-2 \cos \left(2 \pi \phi_{i}\right)\right)
$$

Taylor expansion of this potential leads immediately to the F-term part of (3.4).

The potential (3.10) can be usefully rewritten in a more general form

$$
V=V_{0}+\sum_{i} \alpha_{i} \cos \left(2 \pi \phi_{i}\right)+\sum_{i, j} \beta_{i j} \cos \left(2 \pi \phi_{i}-2 \pi \phi_{j}\right)
$$

from which we can recover (3.10) if we take the obvious definitions of the axion-independent term $V_{0}$ and of the coefficients $\alpha_{i}, \beta_{i j}$.

The simplest sort of N-axion potential would have been a sum of cosine potentials, each involving a single field, with negligible cross-couplings, i.e. $\beta_{i j}=0$. We have just seen that such a simple potential never governs deviations from an approximately F-flat KKLT minimum. While we do not claim that uncoupled $\mathrm{N}$-axion potentials are impossible in string constructions, we do expect that they are rather rare in general. It would be very interesting to find exceptions to this rule.

Requiring that the $\beta_{i j}$ are negligibly small is also a very significant constraint on the general form (3.11). The problem is that there are $N^{2}$ cross-coupling terms but only $N$ single-field terms, so cross-couplings which appear small could still be important in large

12 The terms from $-3|W|^{2}$ have an analogous form but complicate the formulas substantially. Because these terms are unimportant for our considerations, we omit them here. 
numbers. (However, as explained in [1], the fact that each field appears in only a small fraction of the couplings suggests that assisted inflation is still possible. See the Appendices for a more detailed discussion of these issues.)

In our particular case (3.10), it is apparent that the net effect of the terms coupling two axions is not subleading to the effect of the terms involving a single axion. For example, when all the axions are near the origin $\phi_{i}=0$, the two classes contribute amounts of energy that differ only by a factor of two. If instead the axions have a common vev $\bar{\phi} \sim \frac{\pi}{2}$, the cross-terms give the dominant contribution to the potential. Moreover, the energy from these cross-terms is positive and acts to repel the axions from the same point of their configuration space, substantially complicating the motion of the axions. The identification of a single direction for collective motion (such the inflaton $\rho$ of [1]) could be challenging. We will leave the full dynamics of the $\mathrm{N}$-axion potential (3.11) as an interesting problem for the future.

Finally, it is important to observe that diagonalizing the mass matrix is not equivalent to sending $\beta_{i j} \rightarrow 0$ : the presence of cross-couplings ensures that the eigenvalues of $M_{i j}$ are different from the $\alpha_{i}$. The correct procedure, when the quadratic approximation is applicable, is to rotate away the cross-couplings, not to omit them. Surprisingly, this simplifies the problem of understanding the mass spectrum: we will see that although the collections $\left\{\alpha_{i}\right\}$ vary considerably from one compactification to another, the statistical properties of the spectrum of eigenvalues $\left\{m_{i}^{2}\right\}$ of $M_{i j}$ are much less variable.

\section{Random Matrix Treatment of the Mass Terms}

In order to understand which classes of N-flation models are most likely, we must understand the properties of the mass matrix $M_{i j}$, (3.9). However, the complete expression is prohibitively complicated, particularly because it depends on the unknown quantities $D_{A} C_{i}$. For this reason, we resort to characterizing the expected properties of the ensemble $\Omega_{M}$ of mass matrices one expects in an ensemble of N-flation models. More specifically, we seek the probability distribution function $p\left(m^{2}\right)$ for the eigenvalues $m_{i}^{2}$ of the mass matrix, a function which we call the mass spectrum for short.

The logic behind this step is that, even without knowing the individual terms $D_{A} C_{i}$, we can learn something about the eigenvalues of the mass matrix by searching for properties of the distribution of eigenvalues of (3.9) that are independent of the particular set of values taken on by the $D_{A} C_{i}$ in any given realization. This approach is effective because 
the mass matrices are so large that fluctuations away from the mean behavior are unlikely. Thus, knowledge about average elements of $\Omega_{M}$ provides an excellent guide to the properties of nearly all the members of the ensemble. We will therefore restrict ourselves to characterizing $\Omega_{M}$ as a whole.

Moreover, although we will be able to establish some facts about the statistics of the $D_{A} C_{i}$ themselves, 13 it is possible, and advantageous, to find properties of the eigenvalue spectrum that are substantially independent not just of the particular values attained by the $D_{A} C_{i}$, but even of the distribution $\Omega_{D}$ of these values in an ensemble of models. Because the matrices in question are moderately large, simplifications of the sort that often arise at large $N$ in random matrix theory turn out to simplify the problem. A particular result [27] which provides a strong motivation for our approach is this: the behavior of the eigenvalue spectra of $N \times N$ random matrices whose entries have appropriately bounded moments but an otherwise arbitrary distribution $\Omega_{D}$, is identical, in the $N \rightarrow \infty$ limit, to the spectrum in the simplest case in which the entries are independent and identically distributed (henceforth i.i.d.) and $\Omega_{D}$ is a Gaussian distribution with mean zero. This is an extremely powerful statement, and although we will not appeal to it directly, it gives an underlying reason that one might have expected universal behavior in our system.

\subsection{Statistical Model for the Covariant Derivatives}

In the basis in which the kinetic terms are canonical, the mass matrix takes the form (3.9). The mass terms arising from $-3|W|^{2}$ are subleading, by a factor of the inverse volume $\frac{1}{\rho}$, to those coming from the F-terms. For this reason, we neglect the former in the analytical discussions that follow. In $§ 5.2$ we will present the results of a Monte Carlo simulation of the full structure (3.9) to show that this approximation is a sound one. There we will find that even without including the suppression by $\frac{1}{\rho}$, the terms from $-3|W|^{2}$ have a negligible effect on the statistical properties of the mass matrix.

The remaining quantities of interest are the rotation matrices $O_{i}{ }^{j}$, the decay constants $f_{i}$, and, most importantly, $H_{A i} \equiv D_{A} W_{i}$, the $(N+P) \times N$ matrix of Kähler-covariant derivatives.

We will now attempt to characterize the statistical properties of the entries in $H$. We propose that the entries of $H$ may be modeled as independent, identically distributed

13 More sophisticated treatments of the statistics of flux vacua appear in [36. 
(i.i.d.) variables with mean $\mu_{H}$ and variance $\sigma_{H}^{2}$. We do not assume that the distribution is Gaussian.

From these assumptions, one can show that in fact $\mu_{H} \ll \sigma_{H}$. Observe that the row-average $r_{A} \equiv \frac{1}{N} \sum_{i} H_{A i}=-\frac{1}{N} D_{A} W_{0}$ by the F-flatness condition. Granting the i.i.d. assumption stated above, the Central Limit Theorem implies that the row-averages $r_{A}$ are drawn from a distribution with mean $\mu_{H}$ and standard deviation $\frac{\sigma_{H}}{\sqrt{N}}$. The sign of every $r_{A}$ is therefore the sign of $\mu_{H}$, unless $\sigma_{H} \gtrsim \sqrt{N} \mu_{H}$ (so that a fluctuation can change the sign of one or more of the $R_{A}$.)

However, genericity and microscopic considerations require that the terms $r_{A}=$ $-\frac{1}{N} D_{A} W_{0}$ take on both signs. In particular, the sign of $r_{i}=-\frac{1}{N} W_{0} \partial_{i} K$ depends on the intersection numbers $C_{i j k}$ via $\partial_{i} K=-\frac{1}{\mathcal{V}} C_{i j k} t^{j} t^{k}$. N-flation is only possible in compactifications in which some fraction of the $C_{i j k}$ are negative (in order to avoid excessive renormalization of Newton's constant [1]), so the $r_{A}$ cannot all have the same sign. If our distribution is to reproduce this basic feature, the mean value $\mu_{H}$ of the $H_{A i}$ must be taken to be parametrically small compared to the standard deviation,

$$
\mu_{H} \lesssim \frac{\sigma_{H}}{\sqrt{N}}
$$

For all practical purposes we may therefore set $\mu_{H}=0.14$

Now define

$$
R_{A i}=2 \pi e^{K / 2} f_{i}^{-1} O_{i}^{j} H_{A j}
$$

so that the leading contribution to the mass matrix is

$$
M_{i j}=R_{i A} R_{j}^{A}
$$

Provided that the rotations $O$ are random, it follows from $\mu_{H}=0$ that the mean $\mu$ of the $R_{A i}$ may likewise be approximated by zero. However, the variance $\sigma^{2}$ of the $R_{A i}$ may be different from $\sigma_{H}^{2}$.

In summary, we claim that for the purpose of determining the spectrum, the essential structure of the mass matrix is (4.3), where $R_{A i}$ is the $(N+P) \times N$ matrix defined in (4.2). We further propose to approximate the entries of $R$ as i.i.d. variables with zero mean and some variance $\sigma^{2}$. We repeat that the entries do not necessarily have a Gaussian

14 Even if an exception to this result could be found, the addition of a mean $\mu_{H} \neq 0$ would not change the spectrum of masses of the $N-1$ lightest axions [27]. 
distribution. To underscore both this point and the results of $\S 4.1$, we will construct, in $\S 5$, ensembles of random mass matrices using the full formula (3.9). For a range of assumptions about the distributions of the entries of $K^{A B}$ and $H_{A i}$, we will find results indistinguishable from those that follow from (4.3).

We have not found an exact expression for $\sigma$ in terms of microscopic parameters, beyond the definition

$$
\sigma \equiv\left\langle\left|R_{A i}\right|\right\rangle
$$

where the average is over all values of the indices $A, i$. However, a useful heuristic is

$$
\sigma^{2} \sim \frac{e^{K}}{\left\langle f^{2}\right\rangle}\left\langle\left(D_{A} C_{i}\right)^{2}\right\rangle
$$

which shows that $\sigma$ measures the typical size of the F-terms. The scale of the total superpotential, and thus of the F-terms, is determined by size of the flux-induced superpotential $W_{0}$, which in turn is fixed by a choice of fluxes. As in other applications of the KKLT proposal, a fine-tuning of the value of $W_{0}$ is necessary in order to construct a vacuum at large volume and weak coupling. Although many moduli are present in our setup, a single fine-tuning, that of $W_{0}$, suffices to fix the overall scale, and hence the typical axion mass $\bar{m}$. In practical terms, we set the value $\bar{m}$ exactly as in [1], by requiring that the density perturbations resulting from inflation have amplitude consistent with observations. This implies (see $\S 8)$

$$
\bar{m} \approx 1.5 \times 10^{-5} M_{p}
$$

Let us point out that (4.6) is not satisfied by an $\mathrm{N}$-axion potential in a truly generic string compactification, which would presumably have an average mass of order one in string units. We must restrict our attention to the subclass of models (with fine-tuned fluxes) in which the overall scale does obey (4.6). Of course, this problem is not special to N-flation: in nearly every model of inflation in field theory or string theory, one must set the scale of inflation by hand. In our case, the necessary fine-tuning happens to be of the form treated by Bousso and Polchinski [37].

The distribution of the axion masses around the average scale (4.6) is the quantity that we will now determine with random matrix techniques. 


\subsection{Axion Masses and the Marčenko-Pastur Law}

From the complicated structure of the mass matrix (3.9) we have abstracted one essential property:

$$
M=R^{T} R
$$

where $R$ is a $(N+P) \times N$ rectangular matrix whose entries are i.i.d. with zero mean and variance $\sigma^{2}$. We have therefore reduced the problem of finding the mass spectrum in a typical $\mathrm{N}$-flation model to that of finding the eigenvalue probability distribution function

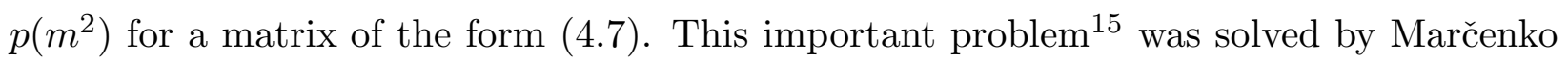
and Pastur in 1967 [39].

The Marčenko-Pastur law for the eigenvalue spectrum is

$$
p\left(m^{2}\right)=\frac{1}{2 \pi m^{2} \beta \sigma^{2}} \sqrt{\left(b-m^{2}\right)\left(m^{2}-a\right)}
$$

for $a \leq m^{2} \leq b$, where we have defined

$$
\begin{gathered}
a=\sigma^{2}(1-\sqrt{\beta})^{2} \\
b=\sigma^{2}(1+\sqrt{\beta})^{2} \\
\beta=\frac{N}{N+P}
\end{gathered}
$$

The probability density vanishes outside this range. In Fig.1 we display the MarčenkoPastur law for a few illustrative cases.

The parameters controlling the shape of the spectrum (4.8) are $\beta$ and $\sigma$, where $\beta$ is the ratio of the dimensions of the rectangular matrix $R$ and $\sigma^{2}$ is the variance of the entries of $R_{A i}$. In the case of a N-flation in a KKLT compactification of type IIB string theory, there are $h_{1,1}$ axions, and $h_{1,1}+h_{2,1}+1$ is the total dimension of the moduli space (Kähler, complex structure, and dilaton), so that

$$
\beta=\frac{h_{1,1}}{h_{1,1}+h_{2,1}+1}
$$

15 The problem of finding $p(\lambda)$ for an ensemble of matrices of the form (4.7) is important for statistical analysis and also has a range of practical applications, for example in communications engineering [38]. Here we denote the eigenvalue by $\lambda$ because the interpretation of an eigenvalue as a mass-squared is rare in these other applications. 


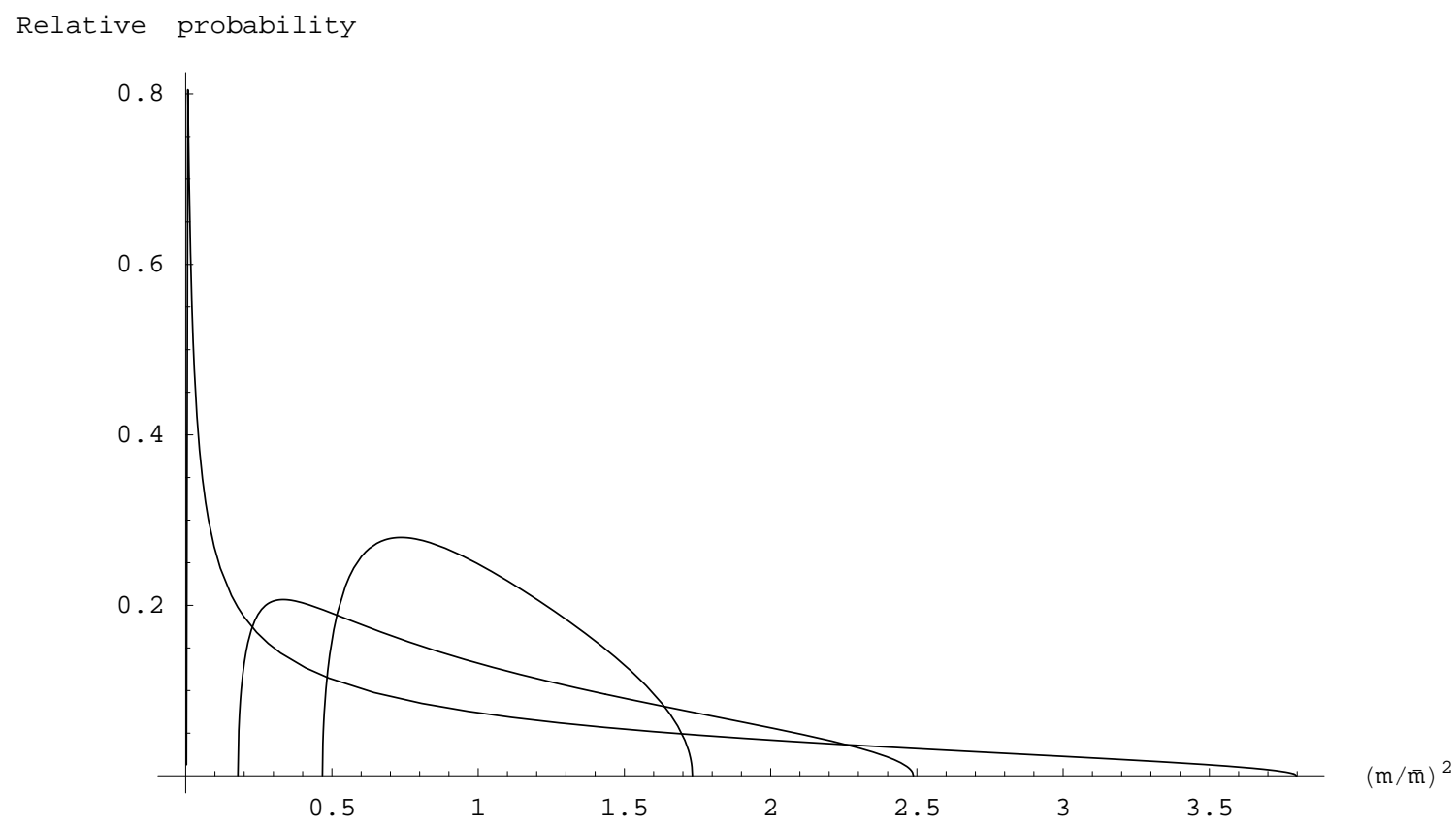

Fig. 1: The Marčenko-Pastur law for $\beta=9 / 10$ (highest peak), $1 / 3,1 / 10$ (most localized).

In more general cases, for example in other string theories, it may be possible to identify $\beta$ with a ratio of this form: the number of (real) axions involved in inflation divided by the total number of moduli chiral multiplets.

We will not attempt to find the smallest and largest values of $\beta$ that can arise in a working N-flation model. In any case, there is an important, independent constraint on $\beta$. The renormalization of Newton's constant described in [1] and reviewed in $\S 9.1$ requires that $N \sim P$, so that $\beta \sim \frac{1}{2}$. In special (and perhaps fine-tuned) circumstances this constraint might be relaxed, so we perform the remainder of our analysis for a general $\beta$. However, we will keep in mind that models with $\beta \sim \frac{1}{2}$ are strongly favored because they more readily produce an appropriate number of e-folds.

To determine the value of $\sigma$, notice from (4.8) that the average value of the masssquared is

$$
\left\langle m^{2}\right\rangle=\sigma^{2}
$$

from which it is evident that $\sigma$ sets the overall mass scale. As explained in the previous section, the only phenomenologically viable N-flation models are those in which (4.6) is satisfied. This effectively fixes the value of $\sigma$ and leaves $\beta$ as the unique parameter specifying an N-flation model. 


\subsection{Some Relations to Other Results in Random Matrix Theory}

The Marčenko-Pastur law [39] is in some sense analogous to Wigner's celebrated semicircle law 25] for the distribution of eigenvalues of a random symmetric matrix $M=H+H^{T}$, where the entries of the square matrix $H$ are i.i.d. Gaussian variables with mean zero. In the same way, $\Omega_{M}$ has some superficial similarities to the famous Gaussian Orthogonal Ensemble (GOE), which describes symmetric matrices whose entries are drawn from normal distributions.

Recall that elements of the GOE [24] may be constructed as $G=H+H^{T}$, where the entries of $H$ are i.i.d. Gaussian variables with zero mean. The eigenvalues of $G$ obey Wigner's semicircle law. Our mass matrix has instead the structure $M=H^{T} H$, and substantial correlations among the matrix entries in $M$ conspire to give the MarčenkoPastur spectrum of eigenvalues.

The Marčenko-Pastur law (4.8) does approach a semicircle law, although not one centered at the origin, in the limit $\beta \rightarrow 0$. This limit corresponds to $P / N \rightarrow \infty$, so that the number of axions is very small compared to the total number of moduli. Compactification manifolds in which $P \gg N$ and $N \gg 1$ are presumably quite rare, so we find that the Marčenko-Pastur law is quite different from the semicircle law for realistic values of $\beta$.

In the other limiting case $\beta \rightarrow 1$, the Marčenko-Pastur spectrum approaches the square of a Wigner semicircle spectrum (also known as the quarter-circle law [26]); that

is, one can construct the former spectrum by squaring the eigenvalues appearing in the latter. Squaring a symmetric but otherwise general matrix $H$ is one way to construct a matrix which is positive-definite (as we have demanded that our mass matrix should be) and has an easily-computed spectrum. In the limit $\beta \rightarrow 1$, in which there are very few chiral multiplets in the theory except for those in which the axions appear, the N-flation mass spectrum actually arises in this very simple way.

In the special case that the distribution of the $R_{A i}$ is Gaussian, $M$ is actually a real Wishart matrix [40], about which a great deal is known. In this paper we do not assume that the distribution is Gaussian, as there is no clear physical motivation for this beyond the universal behavior of random matrices [27] in the $N \rightarrow \infty$ limit. However, in the event that exact results are desired for a toy model, the Wishart ensemble could prove to be quite tractable. 


\section{Monte Carlo Analysis of Random Mass Matrices}

In the preceding section we made a few assumptions about the statistical properties of the Kähler-covariant derivatives $D_{A} C_{i}$ and about the Kähler metric itself. Moreover, we argued that the essential structure of the mass matrix (3.5) is captured by (4.7). These simplifications led to a universal result for the mass spectrum, the Marčenko-Pastur law (4.8).

In this section we use Monte Carlo methods to provide further evidence for the conclusions of $\S 4$. In particular, we show that the results are robust against relaxation of our assumptions. We construct a collection of mass matrices according to the full rule (3.5), using a variety of input distributions for $D_{A} C_{i}, K^{A B}$. The result is indistinguishable from (4.8), providing a strong confirmation our analytic result.

To generate a random mass matrix $M_{i j}$, we choose the entries of the component matrices $K^{A B}, C_{i} C_{j}$, and $D_{A} C_{i}$ from appropriate ensembles $\Omega_{K}, \Omega_{C}, \Omega_{H}$. This procedure is clearly superior to postulating the distribution $\Omega_{M}$ of the entries of $M_{i j}$ directly, because the latter procedure produces no new information. In contrast, assembling the component matrices according to (3.5) may be expected to yield results that are substantially independent of the (largely unknown) details of $\Omega_{K}, \Omega_{C}, \Omega_{H}$, but that do depend on the known structure of the matrix products in (3.5). We will see that this expectation is justified.

\subsection{Properties of the Component Matrices}

Although the Kähler metric is determined by the intersection numbers $C_{i j k}$ of the Calabi-Yau, the scarcity of examples where all intersection numbers are known makes this an impractical route to an understanding of the properties of a typical $K^{i j}$. For this reason, our examples of "Kähler metrics" were simply random, symmetric, positive-definite matrices.

The derivatives $D_{A} C_{i}$ split into two classes. For $A=a$, i.e. when the derivative is with respect to a complex structure modulus, we have

$$
D_{a} C_{i}=\partial_{a} K C_{i}+\partial_{a} C_{i}
$$

and we recall that $C_{i}$ may depend on the complex structure sector through the prefactor $A$, which is a fluctuation determinant in the case that the nonperturbative superpotential comes from Euclidean D3-branes. As we have stressed, the form of this dependence for a general threefold is not known. 
For $A=i$, i.e. in the Kähler sector, we have instead

$$
D_{i} C_{j}=\partial_{i} K C_{j}-2 \pi C_{j} \delta_{i j}
$$

so that the average behavior of the diagonal elements is distinguishable from that of the off-diagonal elements.

Observing that each of the three classes of individual terms $\partial_{a} C_{i}, \partial_{A} K, C_{i}$ can be positive or negative, we constructed samples of $D_{A} C_{i}$ by drawing these terms from distributions that ranged over both positive and negative numbers of order one. We found that for a wide range of properties of these distributions, the mean value $\mu$ of the elements of the resulting matrices $D_{A} C_{i}$ is much smaller than the standard deviation $\sigma$. This is consistent with our analytic demonstration, in $\S 4.1$, that the mean value of these elements is necessarily small compared to their standard deviation.

\subsection{Results of Monte Carlo Simulation}

We generated thousands of examples of mass matrices using the rules outlined in the previous section. In particular, we varied the distributions $\Omega_{K}, \Omega_{C}, \Omega_{H}$. For example, in the case of $\Omega_{H}$ we considered normal distributions with various values of the mean; uniform distributions; log-normal distributions; chi and chi-square distributions; and many other distributions assembled from these via matrix products.

The results of this simulation are as follows. The distribution of matrix elements is bimodal, with the off-diagonal entries normally distributed around zero, and the diagonal entries normally distributed around a positive mean. As may be expected from the central limit theorem, the simulated distributions are extremely close to normal distributions for a wide range of input parameters.

The positive mean of the diagonal terms is large compared to the expected magnitude of the off-diagonal terms. Specifically, we find that the typical magnitude of off-diagonal elements is smaller than the typical magnitude of diagonal elements by a factor

$$
\varepsilon=\frac{1}{\sqrt{N+P}}
$$

The numerical coefficient is of order one. This suppression is of limited relevance for our primary goal of understanding the mass spectrum, but it could be useful in other contexts, such as an analysis of the full axion potential (3.10). For this reason, we provide an analytic derivation of this suppression factor in Appendix A. 
Finally, and most importantly, the eigenvalues are indeed distributed according to the Marčenko-Pastur law, as shown in Fig. 2. The shapes of the empirical eigenvalue distributions agree extremely well with those predicted by (4.8), without any need for tuning or fitting of parameters.

The spectra shown are for the simplest case, that of a Gaussian distribution with mean zero. In each of the other cases we tested, the spectra are identical to those shown, except for the presence of a single eigenvalue $N$ times larger than the others. The presence of this single large eigenvalue is familiar from random matrix theory [27]. In physical terms, the associated field will rapidly move to its minimum and decouple from the inflationary dynamics. Because a spectrum with an extreme outlier is not easily interpreted visually, we do not show an example.

\section{N-flation Dynamics and Initial Conditions}

Having derived the mass spectrum for the fields contributing to N-flation, we are now in a position to evaluate the cosmological evolution associated with this system.

\subsection{Inflationary Dynamics}

As usual, the dynamics are described by the general set of equations,

$$
\begin{gathered}
H^{2}=\frac{1}{3 M_{P}^{2}}\left[\frac{1}{2} \sum_{i} \dot{\phi}_{i}{ }^{2}+V(\phi)\right] \\
\dot{H}=-\frac{1}{2 M_{P}^{2}} \sum_{i} \dot{\phi}_{i}{ }^{2} \\
\ddot{\phi}_{i}+3 H \dot{\phi}_{i}+\frac{\partial V}{\partial \phi_{i}}=0
\end{gathered}
$$

We will make frequent use of the fact that our potential $V$ is written as a sum over terms of the form $m_{i}^{2} \phi_{i}^{2} / 2$.

During slow roll, one can find a general result for the field evolution [17]. Dropping the acceleration terms from the fields' equations of motion, and dividing the $\phi_{i}$ equation by the $\phi_{j}$ equation, we see

$$
\frac{\dot{\phi}_{i}}{\dot{\phi}_{j}}=\frac{m_{i}^{2}}{m_{j}^{2}} \frac{\phi_{i}}{\phi_{j}}
$$



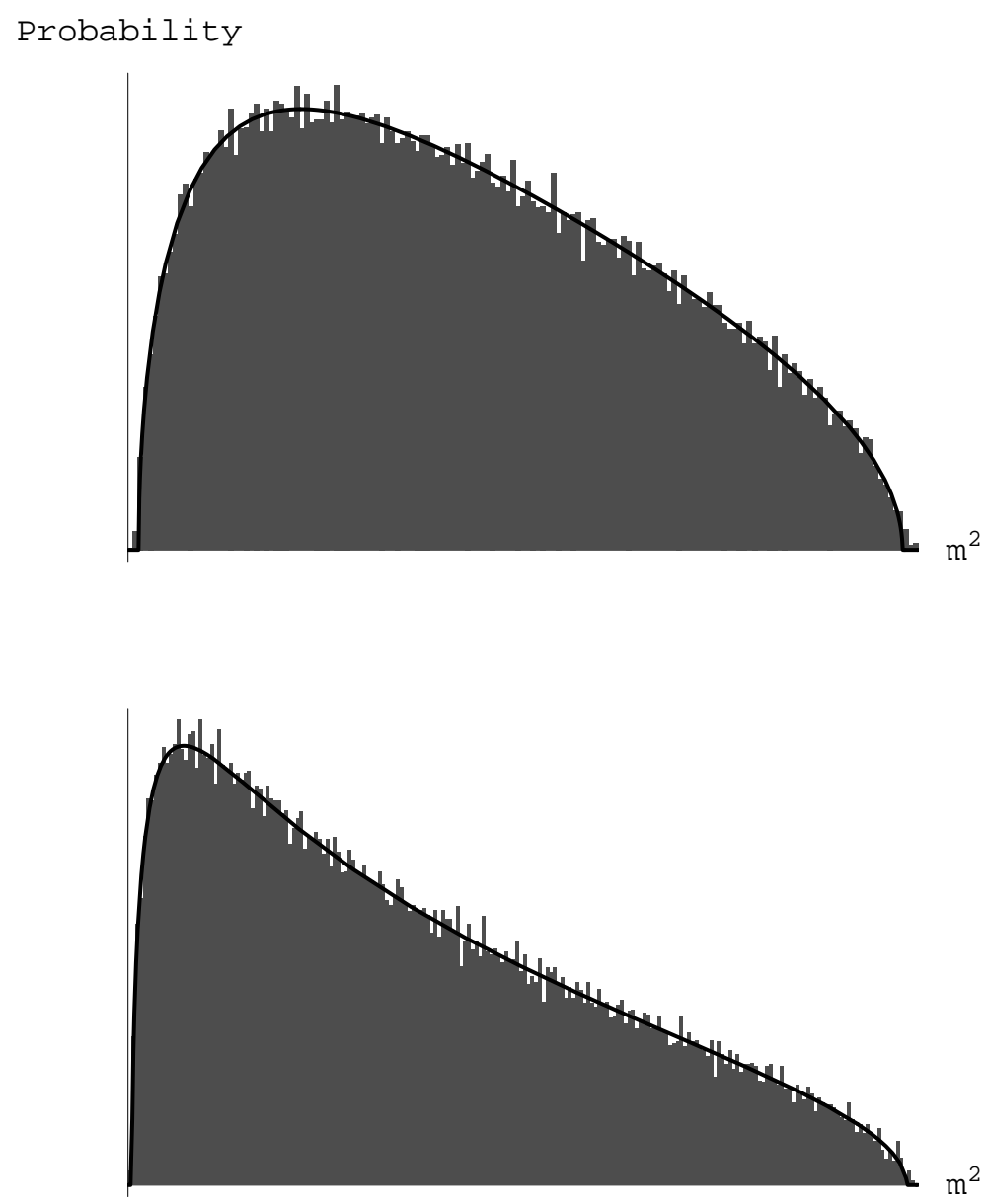

$m^{2}$

Fig. 2: Empirical eigenvalue spectra of 100 random $300 \times 300$ matrices constructed according to (4.7), with $\beta=1 / 10,1 / 3,9 / 10$. The horizontal axis is the mass-squared and the vertical axis is the relative probability. The overlaid curves show the Marčenko-Pastur law in each case. 


$$
\Rightarrow \frac{\phi_{i}(t)}{\phi_{i}\left(t_{0}\right)}=\left(\frac{\phi_{j}(t)}{\phi_{j}\left(t_{0}\right)}\right)^{m_{i}^{2} / m_{j}^{2}}
$$

This is not an attractor: if we rescale the initial value of one of the fields by multiplying $\phi_{i}\left(t_{0}\right)$ by a constant, this rescaling changes the field value at all subsequent times, for as long as the slow roll approximation remains valid.

It is instructive to specialize to the Marčenko-Pastur spectrum, where the lightest field $\phi_{1}$ has $m_{1}^{2} \approx a(4.9)$, the average mass-squared is $\sigma^{2}$, and the most massive field $\phi_{N}$ has $m_{N}^{2} \approx b(4.10)$. Let us use the progress of $\phi_{N}$ as a clock, via

$$
\tau(t) \equiv \frac{\phi_{N}(t)}{\phi_{N}\left(t_{0}\right)}
$$

As inflation proceeds, $\tau$ decreases. Trivially rewriting (6.5), we have

$$
\phi_{i}(t)=\phi_{i}(0) \tau(t)^{Q_{i}}
$$

where $Q_{i}=\frac{m_{i}^{2}}{b}$ are in the range

$$
\frac{a}{b}=\left(\frac{1-\sqrt{\beta}}{1+\sqrt{\beta}}\right)^{2} \leq Q \leq 1
$$

(In the preferred case $\beta \approx \frac{1}{2}$, we have $\frac{a}{b} \approx \frac{1}{34}$.) Even if the fields begin near the same point in field space, they quickly spread out: the large range in the exponents $Q_{i}$ ensures that the fields move at very different rates. We illustrate this spreading in Fig. 3.

If instead each field had a $\lambda_{i} \phi_{i}^{4}$ potential, the solution analogous to (6.5) would be

$$
\frac{1}{\phi_{i}(t)^{2}}-\frac{1}{\phi_{i}\left(t_{0}\right)^{2}}=\frac{\lambda_{i}}{\lambda_{j}}\left(\frac{1}{\phi_{j}(t)^{2}}-\frac{1}{\phi_{j}\left(t_{0}\right)^{2}}\right)
$$

We observe that the initial conditions become increasingly irrelevant at late times, demonstrating the existence of an attractor in the $\lambda \phi^{4}$ case. This is a desirable feature in a model of inflation, but because realizing assisted $\lambda \phi^{4}$ inflation in string theory (or in any well-motivated particle physics setup) is probably very difficult, we will say no more about this case.

The absence of an attractor solution for N-flation significantly complicates the inflationary dynamics. If there were a late-time attractor, and inflation lasted long enough to eliminate the residual effects of the initial conditions, we could assume that the system followed the attractor solution during the last 60 e-folds of inflation, greatly simplifying 


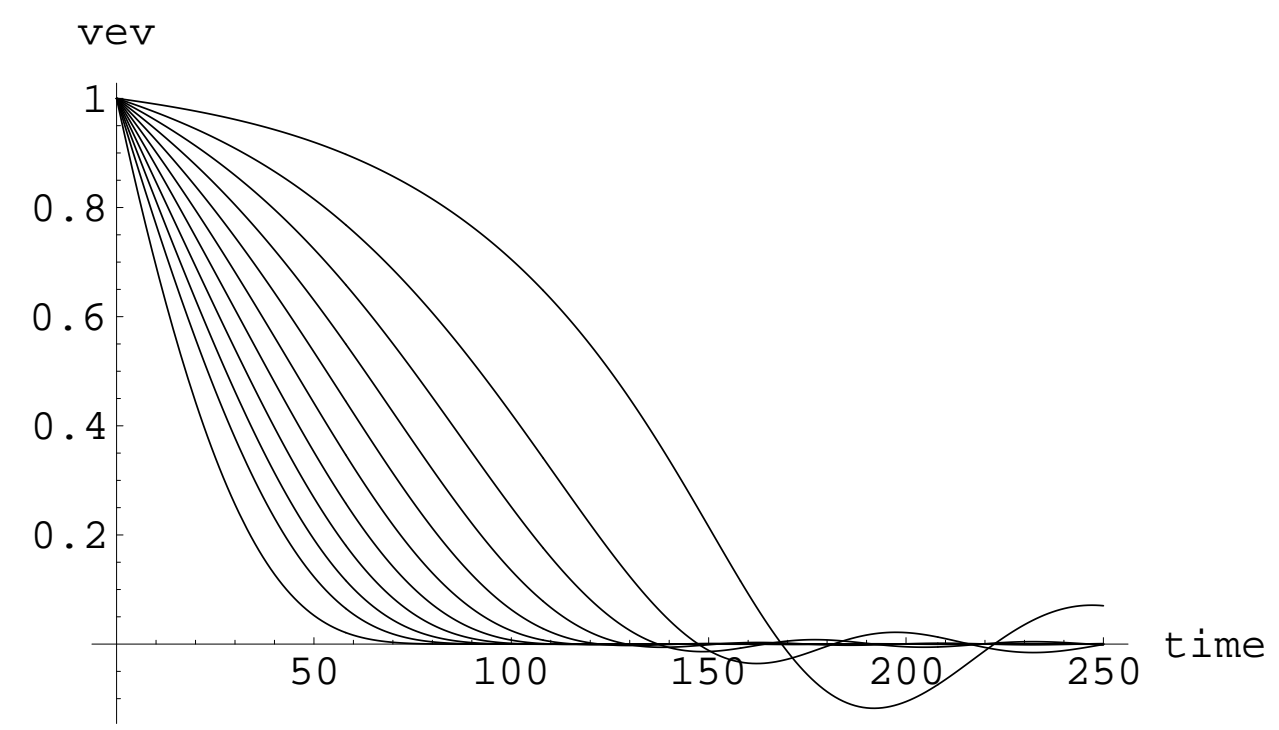

Fig. 3: We plot the evolution of $\phi_{i}$ as a function of time for $N=201$, $\beta=1 / 2$, and $i=1,21, \cdots, 201$ with $\bar{m}=10^{-6} M_{P}$, and time measured in units where $\bar{m}=1$.

the treatment of the perturbations. By contrast, we will see in the next section that the $\mathrm{N}$-flation spectrum is a function of the values of the fields measured at some fiducial time, since the initial conditions never become irrelevant.

Finally, notice that in single-field inflation, the breakdown of slow roll coincides with the end of inflation, and to a first approximation the two events can be regarded as equivalent. In N-flation, however, slow roll can fail well before the end of inflation for fields that are particularly massive or that begin close to the origin.

\subsection{Initial Conditions}

We will not give a full treatment of the initial 19 conditions in this paper. However, a few observations will be essential.

The data of an N-flation model in the four-dimensional effective theory is a spectrum of $N$ axion masses. Given any such system, one would like to know whether the model can produce, with some choice of initial conditions, $\mathcal{N} \gtrsim 60$ e-folds of inflation. For this purpose it is useful to choose initial conditions that maximize the number of e-folds while remaining consistent with all control assumptions, especially the requirement that the largest vev is

16 Throughout this paper, we use "initial" to refer to the situation 60 e-folds before the end of inflation. This is not necessarily the start of cosmic history. 
at most of order $M_{p}$. The amount of inflation is maximized by placing all of the fields as far from the origin as this condition will permit; spreading the initial vevs inevitably moves some fields closer to the origin and thus diminishes the number of e-folds. N-flation systems do not readily produce a vast number of e-folds, so only a tiny fraction of N-flation models can generate enough inflation from a highly "inefficient" initial configuration with disparate vevs. For this reason, we will focus primarily on initial conditions in which the fields are spread by at most an order of magnitude.

Consider any distribution $\left\{\phi_{i}\right\}$ of initial field values, and compute the correlation

$$
\xi \equiv \frac{\left\langle m^{2} \phi^{2}\right\rangle}{\left\langle m^{2}\right\rangle\left\langle\phi^{2}\right\rangle} \equiv \frac{N \sum_{i} m_{i}^{2} \phi_{i}^{2}}{\sum_{j} m_{j}^{2} \sum_{k} \phi_{k}^{2}}
$$

where we have defined

$$
\left\langle m^{p} \phi^{q}\right\rangle \equiv \frac{1}{N} \sum_{i} m_{i}^{p} \phi_{i}^{q}
$$

When $\xi>1(\xi<1)$, we say that the vevs are directly (inversely) correlated with the masses.

We may also parametrize the correlation between masses and vevs as

$$
\phi_{i}=v_{i} m_{i}^{\alpha}
$$

where the variation in prefactors $v$ is presumed to be less important than the dependence on the mass. Let us try to relate $\alpha$ to $\xi$. We begin by observing that for reasonable values of $N,\left\langle m^{2 k}\right\rangle$ is very well approximated as a moment of the Marčenko-Pastur distribution,

$$
\left\langle m^{2 k}\right\rangle \approx m_{(2 k)} \equiv \int m^{2 k} p\left(m^{2}\right)
$$

where the approximation omits terms of order $\frac{1}{N}$,41, which we can safely neglect in the following. The moments of (4.8) are in turn given by a simple analytic formula 42,

$$
m_{(2 k)}=\sigma^{2 k} \sum_{i=1}^{k} \frac{1}{k}\left(\begin{array}{c}
k \\
i
\end{array}\right)\left(\begin{array}{c}
k \\
i-1
\end{array}\right) \beta^{i-1}
$$

We will also need to know $\left\langle m^{-2}\right\rangle$ and $\left\langle m^{-4}\right\rangle$. These are not moments of the distribution, but we can compute them directly from the Marčenko-Pastur law, finding

$$
\left\langle m^{-2}\right\rangle=\frac{1}{\sigma^{2}} \frac{1}{(1-\beta)}
$$


and

$$
\left\langle m^{-4}\right\rangle=\frac{1}{\sigma^{4}} \frac{1}{(1-\beta)^{3}}
$$

Equipped with these results, we can compute $\xi(\alpha)$ in terms of $\beta$ for the cases of interest.

As a consequence of (6.5), the fields rapidly spread apart (except when the masses are degenerate), with the most massive fields relaxing most quickly. This means that the lighter fields soon have larger vevs, on average, than the heavier fields. Thus, positive correlations $\xi \gtrsim 1$ are transient, and inverse correlations $\xi<1$ typically develop. This situation is quite plausible dynamically, but, within the context of controllable N-flation models, it creates a certain tension. Initial conditions with $\xi \gtrsim 1$ are the most efficient at producing many e-folds within the region of control, 17 but because such positive correlations are typically transient, they may not be the most generic initial conditions. We will work not with the initial conditions that are most plausible a priori, but with those that are most plausible given the requirement of $\mathcal{N} \gtrsim 60$ e-folds.

These considerations lead us to focus on initial conditions with $\alpha \approx 0 \Longleftrightarrow \xi \approx 1$, because anything else will typically give inadequate inflation. We will frequently refer to this as an "uncorrelated" initial condition, because the vevs are not correlated to the masses. The equal-field condition $\phi_{i}=\bar{\phi}$ is an important special case of uncorrelated initial conditions. Another instructive configuration, albeit an impractical one for realistic models, is $\alpha=-1$, where each axion makes a roughly equal contribution to the initial energy.

We have numerically evaluated the full equations of motion for a wide variety of parameter values and initial conditions. In Fig. 4 and Fig. 5 we show the results of the evolution for two specific choices of initial conditions: the "equal-field" condition $\alpha=0$, $v_{i} \approx \bar{v}$, and the "equal-energy" condition $\alpha=-1, v_{i} \approx \bar{v}$.

\subsection{Number of E-folds}

We will now study how the amount of inflation depends on $\beta$. Armed with the general result (6.5) for the field evolution, we can write the potential $V(t)$ as an appropriately weighted integral over the Marčenko-Pastur mass distribution, since

$$
V\left(\phi_{N}(t)\right)=\frac{1}{2} \sum_{i} m_{i}^{2} \phi_{i}^{2}\left(t_{0}\right) \tau(t)^{2 Q_{i}}
$$

17 It might be that inflation occurs in nature in a way that is not theoretically computable; for obvious reasons we do not consider this case. 

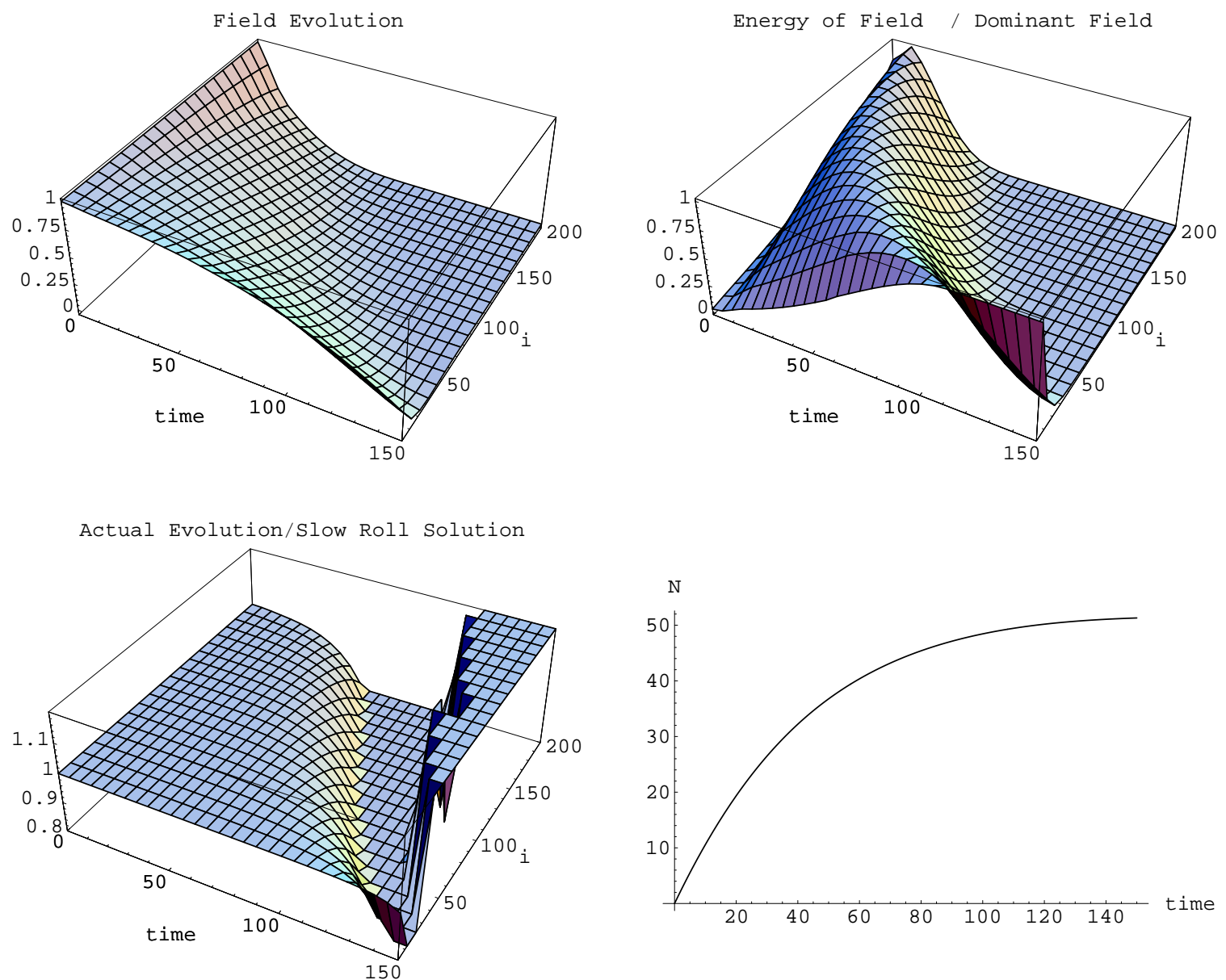

Fig. 4: N-flation dynamics with $N=200, \beta=\frac{1}{2}$, and $\bar{m}=10^{-6} M_{P}$, and equal-field initial conditions. Time is measured in units where $\bar{m}=1$. The fields are arranged along the axis labeled $i$, in order of increasing mass. The top left plot shows the field evolution. The top right plot displays the relative contribution of each field to the overall energy density, normalized by the field that is currently making the dominant contribution. The bottom left plot shows the ratio of the numerical solution to the slow roll solution, (6.5), computed in terms of the vev of the lightest field. The bottom right plot shows the evolution of the scale factor as a function of time. 

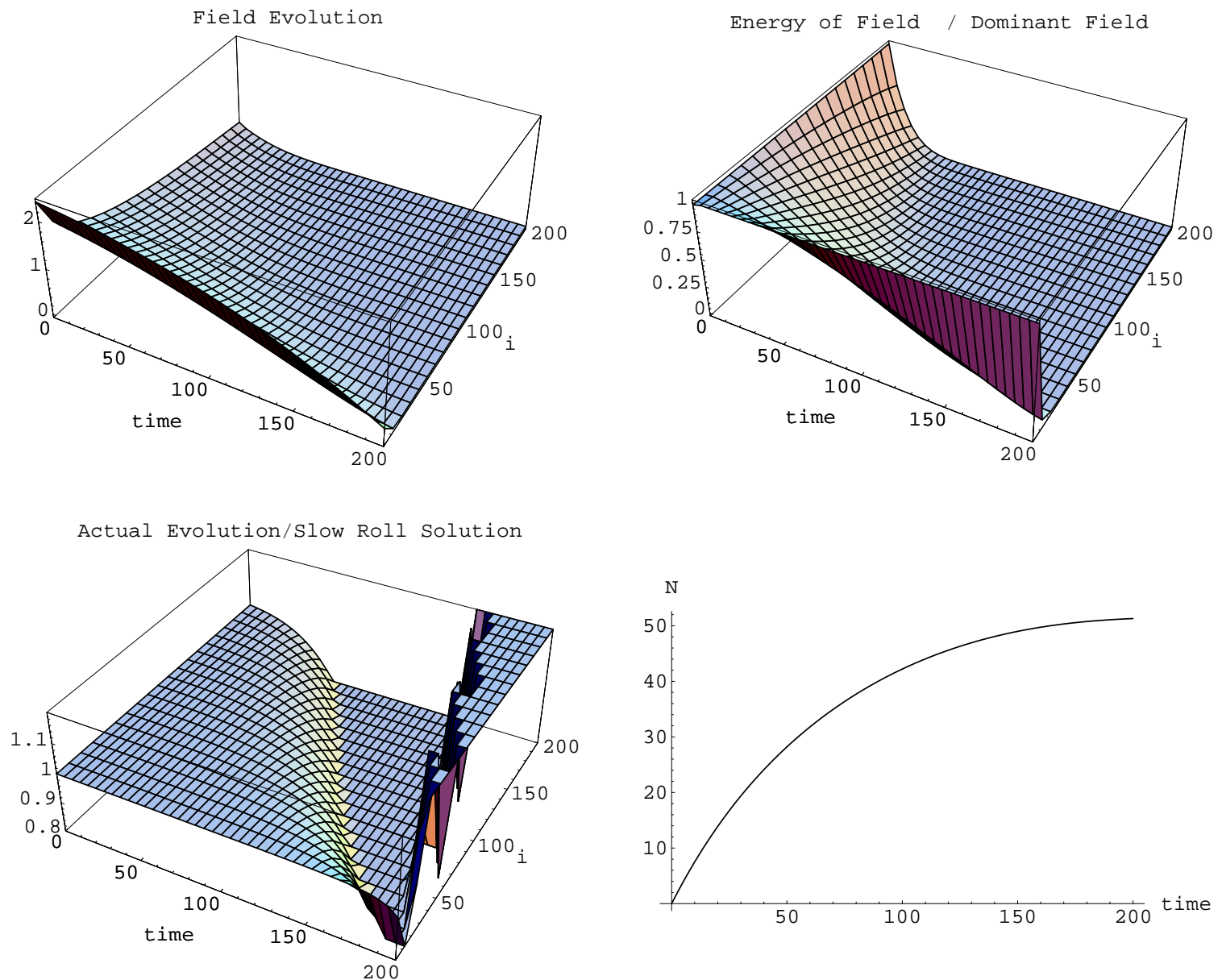

Fig. 5: N-flation dynamics with $N=200, \beta=\frac{1}{2}$, and $\bar{m}=10^{-6} M_{P}$, and equal-energy $(\alpha=-1)$ initial conditions. The plots follow the layout of Figure 4. This is an illustration, not a controllable model: in order for the total number of e-folds to match Figure 4, the initial vevs of the light fields have been taken to exceed $M_{P}$. This is a concrete example of the general observation that for a given mass distribution, initial conditions with $\alpha<0$ require larger maximum vevs in order to produce enough inflation. 
This is approximated by

$$
V(\tau(t))=\frac{\bar{\phi}^{2}}{2} \int_{a}^{b} \lambda x^{\lambda} p(\lambda)
$$

where

$$
x=\tau(t)^{2 / b}
$$

and we have assumed that $\phi_{i}\left(t_{0}\right)=\bar{\phi}$ for all $i$. However, this integral cannot be done in closed form, so we need to make a further approximation in order to compute the number of e-folds of inflation we can expect for a given initial value $\bar{\phi}$. In practice, we are particularly interested in moderate values of $\beta$ - as $\beta$ approaches unity, the mass distribution develops a very long tail, but this situation is disfavored physically. Consequently, we can get a rough estimate of the number of e-folds by assuming that the $N$ fields have equal masses $\bar{m}^{2}=\sigma^{2}$ and possibly different initial vevs $\phi_{i}$. In this case, one can define $\Phi^{2} \equiv N\left\langle\phi^{2}\right\rangle$ and write down an equivalent single-field model [1] with potential

$$
V(\Phi)=\frac{1}{2} \bar{m}^{2} \Phi^{2}
$$

The power of assisted inflation is that each of the $\phi_{i}$ is related to $\Phi$ by $\phi_{i} \approx \Phi / \sqrt{N}$, and while the collective field $\Phi$ must exceed $M_{P}$ by at least an order of magnitude to obtain a workable period of inflation, the vevs of the $\phi_{i}$ can all be sub-Planckian if $N$ is large enough.

The total number of e-folds in this simple model is

$$
\mathcal{N}(\Phi)=\frac{1}{M_{P}^{2}}\left[\frac{\Phi^{2}}{4}-\frac{M_{P}^{2}}{2}\right] \approx \frac{\Phi^{2}}{4 M_{P}^{2}}
$$

This turns out to be an excellent approximation to the number of e-folds produced in the more general case of a spectrum of masses. The quality of this approximation is demonstrated in Figure 6; we conclude that it is safe to ignore $\beta$ when estimating $\mathcal{N}$, unless $\beta$ is very close to unity, and provided that we take initial conditions with approximately equal fields.

The number of e-folds also depends on $\alpha$. As we are physically constrained to consider only sub-Planckian vevs for the $\phi_{i}$ we can compute the maximum number of e-folds by saturating this bound for all the fields. Then any physically realistic initial configuration will have $N$ vevs which are less than or equal to their values in this idealized scenario, and the amount of inflation produced when some vevs are initially reduced from their maximal 


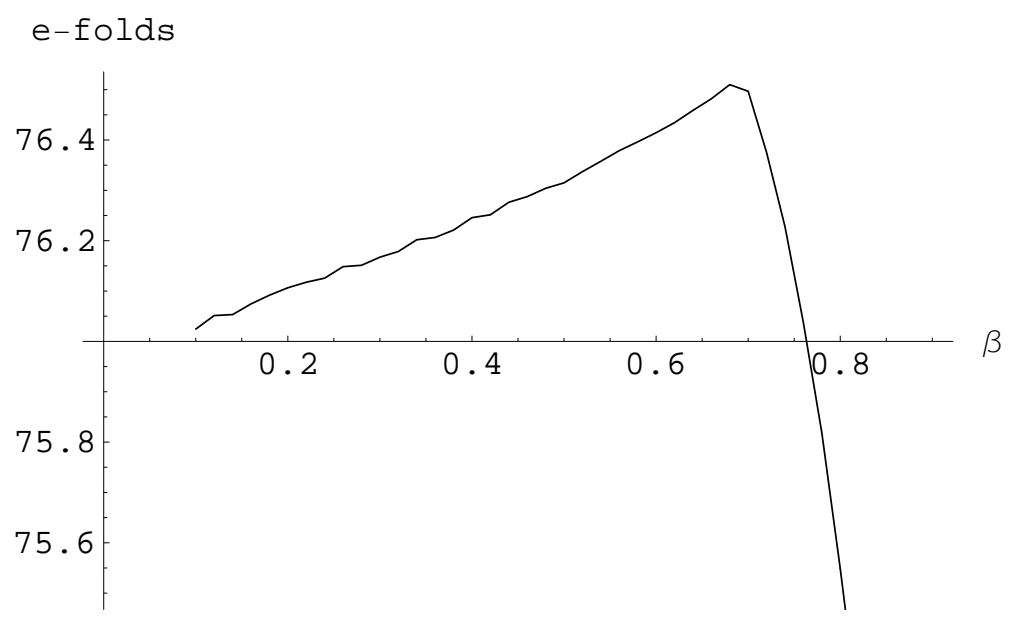

Fig. 6: Numerical results for the number of e-folds $\mathcal{N}$ as a function of $\beta$, with $N=300$ and initial field values $\phi_{i}=M_{P}$. The expected number of e-folds from the approximation in (6.21) is 75 , so when $\beta$ is not very close to unity, the approximation is excellent.

values will necessarily be lower. We can therefore find an upper bound on $\mathcal{N}$ for a given mass distribution by assuming that $\alpha=0$ and $\phi_{i} \approx M_{P}$.

Note that the mass scale $\bar{m}=\sigma$ has fallen out of (6.21). In the end, the mass is fixed not by the number of e-folds, but by the amplitude of the perturbation spectrum, which we now discuss.

\section{Generation of Adiabatic Perturbations}

There is a very general result, derived in [30], for the spectrum of curvature perturbations generated by multi-field inflation,

$$
P_{\mathcal{R}}=\left(\frac{H}{2 \pi}\right)^{2} \frac{\partial \mathcal{N}}{\partial \phi_{i}} \frac{\partial \mathcal{N}}{\partial \phi_{j}} \delta_{i j}
$$

where $\mathcal{N}$ counts the number of e-folds. We will follow the treatment in [10], which specializes (7.1) to the case of uncoupled fields with polynomial potentials,

$$
V(\phi)=\sum_{i} V_{i}\left(\phi_{i}\right)
$$

In the case of N-flation, $V_{i}=\frac{1}{2} m_{i}^{2} \phi_{i}^{2}$. 
The spectrum is given by 10

$$
P_{\mathcal{R}}^{1 / 2}=\sqrt{\frac{V}{12 \pi^{2} M_{P}^{6}} \sum_{i}\left(\frac{V_{i}}{V_{, i}}\right)^{2}}
$$

We have chosen to work with $P_{\mathcal{R}}^{1 / 2}$, since this is proportional to the amplitude of the primordial density perturbations, and to the amplitude of the temperature perturbations in the microwave background. We are implicitly ignoring entropy (isocurvature) fluctuations in this initial analysis, although these are likely to be subdominant for uncoupled fields with very similar decay channels.

For our specific potential, the spectrum becomes

$$
P_{\mathcal{R}}^{1 / 2}=\frac{1}{M_{P}^{3}} \sqrt{\frac{1}{12 \pi^{2}}\left(\sum_{i} \frac{1}{2} m_{i}^{2} \phi_{i}^{2}\right) \sum_{i}\left(\frac{\phi_{i}}{2}\right)^{2}}
$$

Combining these results with (6.11), we find

$$
P_{\mathcal{R}}^{1 / 2}=\frac{N}{M_{P}^{3}} \sqrt{\frac{\left\langle m^{2} \phi^{2}\right\rangle\left\langle\phi^{2}\right\rangle}{96 \pi^{2}}}
$$

We stress that (6.11) is only a convenient notation, and does not imply that an averaging has taken place. The expression (7.5) is exactly equal to (7.4), for any collection of masses and field vevs. Of course, our purpose in writing (7.5) is to make use of statistical information about the masses and initial conditions. To accomplish this, we will now use the moments (6.14) of the Marčenko-Pastur distribution, in combination with various possibilities for the initial conditions.

In the case $\xi=1 \Longleftrightarrow \alpha=0$ that the initial field values are uncorrelated with the masses, which includes the important special case of equal initial vevs, we have

$$
\left\langle m^{p} \phi^{q}\right\rangle=\left\langle m^{p}\right\rangle\left\langle\phi^{q}\right\rangle
$$

so that

$$
P_{\mathcal{R}}^{1 / 2}=\frac{N\left\langle\phi^{2}\right\rangle \sqrt{\left\langle m^{2}\right\rangle}}{\pi \sqrt{96} M_{p}^{3}}
$$

If the masses are taken to be identical, this reduces to the result of an effective single-field model,

$$
P_{\mathcal{R}}^{1 / 2}=\frac{\Phi^{2} m_{\Phi}}{\pi \sqrt{96} M_{p}^{3}}=\frac{V(\Phi)}{m_{\Phi} \pi \sqrt{24} M_{p}^{3}}
$$




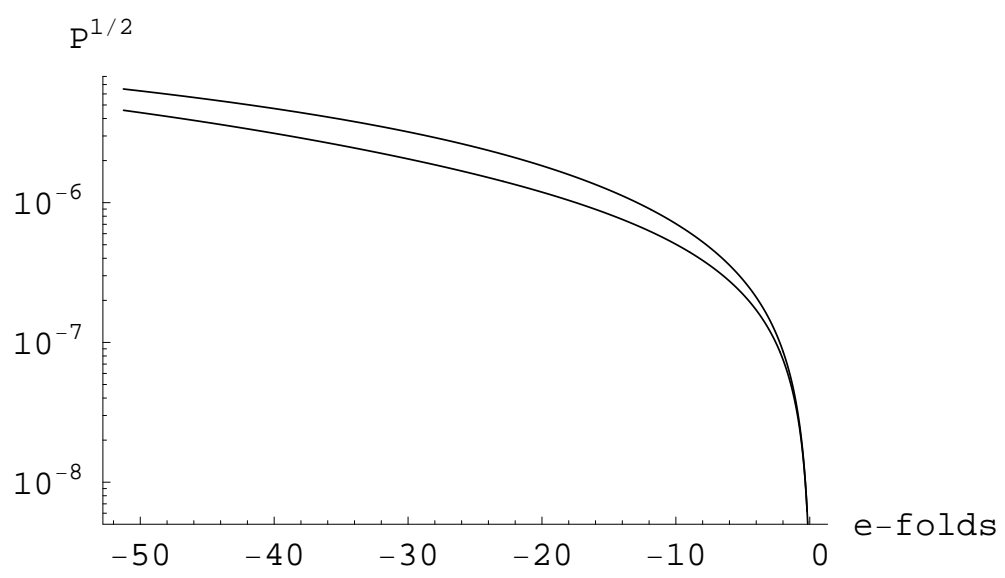

Fig. 7: Scalar power spectra $P_{\mathcal{R}}^{1 / 2}$, computed from (7.4) for the initial conditions used in Fig 4 (lower line) and in Fig 5. The masses are identical in the two cases.

once we identify $\Phi^{2}=N\left\langle\phi^{2}\right\rangle$. This is the simplest possible model of N-flation.

Considering instead the case $\alpha=-1$ in which each field makes a roughly equal contribution to the energy, we find instead

$$
P_{\mathcal{R}}^{1 / 2}=\frac{V}{\bar{m} \pi \sqrt{24} M_{p}^{3}} \frac{1}{\sqrt{1-\beta}}
$$

which is enhanced relative to the single-field result by the modest factor $(1-\beta)^{-1 / 2} \sim \sqrt{2}$.

To get a clearer understanding of the connection between the scale-dependence in the spectrum and the initial field configuration, we now turn to the spectral index $n_{s}$, which is defined as $n_{s}-1 \equiv d \ln P_{\mathcal{R}} / d \ln k$, so that $P_{\mathcal{R}}(k) \sim k^{n_{s}-1}$. For a single inflaton, one has as usual

$$
n_{s}-1=2 \eta-6 \epsilon
$$

where

$$
\epsilon=\frac{M_{P}^{2}}{2}\left(\frac{V^{\prime}}{V}\right)^{2} \quad \eta=M_{P}^{2} \frac{V^{\prime \prime}}{V}
$$

In the case of single-field $m^{2} \phi^{2}$ inflation, this becomes

$$
n_{s}-1=-\frac{8 M_{p}^{2}}{\phi^{2}}
$$

For multiple fields, $n_{s}$ obeys the general formula [30]

$$
n_{s}-1=2 \frac{\dot{H}}{H^{2}}-2 \frac{\mathcal{N}_{, i}\left(\frac{1}{M_{P}^{2}} \frac{\dot{\phi}_{i} \dot{\phi}_{j}}{H^{2}}-M_{P}^{2} \frac{V_{, i j}}{V}\right) \mathcal{N}_{, j}}{\delta_{i j} \mathcal{N}_{, i} \mathcal{N}_{, j}} .
$$


Using once again the specialized results of 10 this reduces to

$$
n_{s}-1=-M_{P}^{2} \sum_{i}\left(\frac{V_{, i}}{V}\right)^{2}-\frac{2 M_{P}^{2}}{\sum_{i}\left(\frac{V_{i}}{V_{, i}}\right)^{2}}+2 \frac{M_{P}^{2}}{V} \frac{\sum_{i} \frac{V_{, i i} V_{i}^{2}}{V_{, i}^{2}}}{\sum_{j}\left(\frac{V_{j}}{V_{, j}}\right)^{2}}
$$

which reverts to (7.12) in the single-field limit. For the special case of multiple quadratic potentials, the final term is proportional to the second term, so

$$
n_{s}-1=-M_{P}^{2} \sum_{i}\left(\frac{V_{, i}}{V}\right)^{2}-\frac{M_{P}^{2}}{\sum_{i}\left(\frac{V_{i}}{V_{, i}}\right)^{2}} .
$$

It follows that $n_{s}-1$ is never positive, and thus that the spectrum is never blue, because we have written $n_{s}-1$ as a combination of squares with a negative overall coefficient.

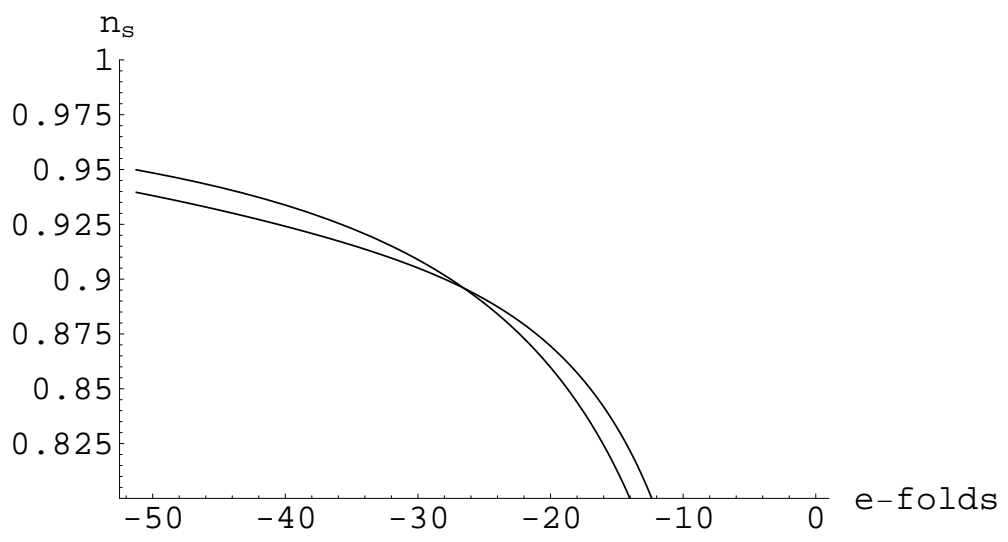

Fig. 8: The spectral indices $n_{s}$ corresponding to the spectra plotted in Fig 7 , displayed as functions of $\log a$. The case with equal initial vevs initially has a higher index.

Using 6.11$)$ we can rewrite $(7.15)$ as

$$
n_{s}-1=-\frac{4 M_{p}^{2}}{N}\left(\frac{1}{\left\langle\phi^{2}\right\rangle}+\frac{\left\langle m^{4} \phi^{2}\right\rangle}{\left\langle m^{2} \phi^{2}\right\rangle^{2}}\right)
$$

Again, we stress that (7.16) follows identically from (7.15), rather than being a statisical approximation. Results that do use statistical reasoning are easily noticed because

18 This simplification is only possible when all the fields are still rolling slowly. 
they contain factors of $\beta$, the parameter that encapsulates the form of a typical mass distribution.

For the case $\alpha=0$ of initial conditions that are uncorrelated with the field masses, we use $(7.6)$ to find

$$
n_{s}-1=-\frac{8 M_{p}^{2}}{N\left\langle\phi^{2}\right\rangle}\left(1+\frac{\beta}{2}\right)
$$

This coincides with (7.12) only in the degenerate limit $\beta \rightarrow 0$. Thus, for this important class of initial conditions, the generalized N-flation spectrum is more red (i.e. further from scale-invariant) than the single-field spectrum. This is one of our main results.

In Fig. 9 we show the $\beta$-dependence of the spectrum (7.17) in the case $\alpha=0$. Because the result shown is the spectral index more than 60 e-folds before the end of inflation, the values shown are closer to scale-invariant than in observationally-relevant cases. Our purpose in showing the figure is establishing the perfect agreement between (7.17) and the result of the numerical evaluation of $(7.15)$.

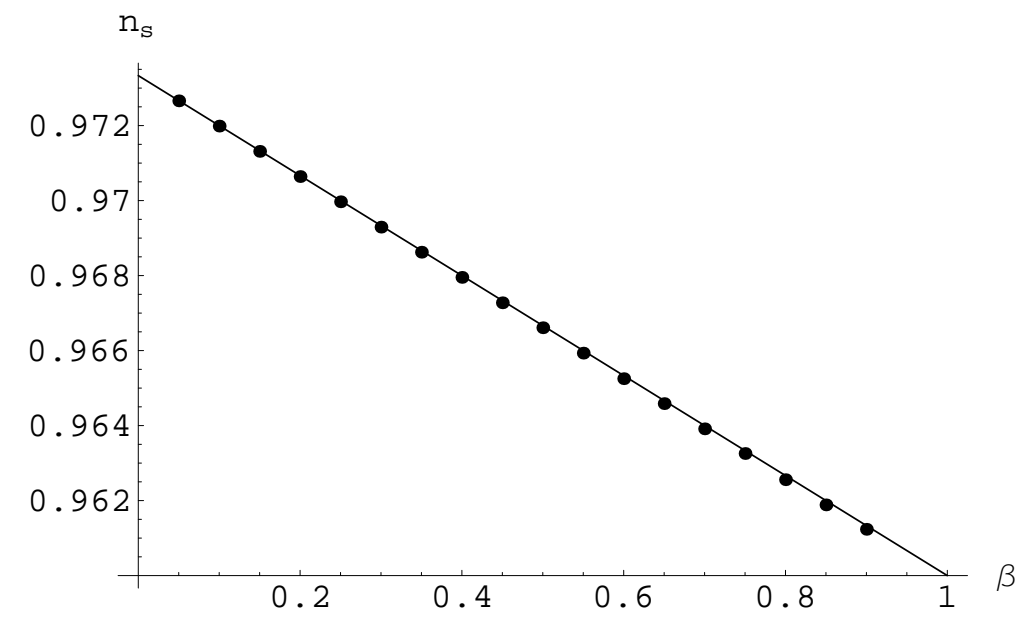

Fig. 9: The initial value of the spectral index as a function of $\beta$, with all $N=300$ fields initially equal to $M_{P}$. The solid line is the theoretical curve (7.17), and the dots come from numerical evaluations of (7.15).

The quantitative relationship between $n_{s}$ and $\beta$ depends on the initial conditions, and in particular on the correlation $\alpha$ between the masses and the initial vevs. As we stressed in $\S 6.2$, the displacements might become correlated inversely with the masses, because the more massive fields relax more rapidly. It would be very interesting to undertake a careful study of the initial conditions for N-flation, and to extract $n_{s}$ as a function of $\alpha$ and $\beta$. However, this is a problem beyond the scope of the present paper. 
Finally, we can compute the running $d n_{s} / d \ln k$ of the scalar power spectrum, by applying the above methods to the general formula given in [10]. The leading contribution is

$$
\frac{d n_{s}}{d \ln k}=-\frac{16}{N^{2}}\left(2 \frac{\left\langle m^{4} \phi^{2}\right\rangle^{2}}{\left\langle m^{2} \phi^{2}\right\rangle^{4}}-\frac{\left\langle m^{6} \phi^{2}\right\rangle}{\left\langle m^{2} \phi^{2}\right\rangle^{3}}+\frac{1}{\left\langle\phi^{2}\right\rangle^{2}}\right)
$$

For the uncorrelated initial condition $\alpha=0$, this simplifies to

$$
\frac{d n_{s}}{d \ln k}=-\frac{16 M_{p}^{4}}{N^{2}\left\langle\phi^{2}\right\rangle^{2}}\left(2+\beta+\beta^{2}\right)
$$

This reproduces the single-field result if we again identify $\Phi^{2}=N\left\langle\phi^{2}\right\rangle$ and send $\beta \rightarrow 0$. Evidently the Marčenko-Pastur spread in masses creates a modest increase in the running, by a factor that is at most 2 and is typically around $\frac{11}{8}$.

This analysis is far from being the last word in understanding the inflationary phenomenology of N-flation. To fully understand the spectrum arising from an arbitrary set of initial conditions, it will be necessary to go beyond first order in slow roll - and perhaps to numerically solve the evolution equations for the field perturbations, since the heavy fields are not well described by the slow-roll approximation during the last e-folds of inflation. Moreover, because there is no attractor solution and the spectrum is a function of the initial field configuration, a solid prediction for the spectral index will require an understanding of the expected distribution of initial values for the axion fields. A further complication we do not consider is that the value of $H$ at the end of inflation depends both on $\beta$ and on the initial values of the axion vevs. In general, increasing $\beta$ while holding the vevs and the overall mass scale fixed will lower the value of $H$ at the end of inflation. This effect must be incorporated in a full calculation of the perturbation spectrum, as it changes the number of e-folds before the end of inflation at which a given comoving scale leaves the horizon, modifying the predicted spectral amplitude for that mode. Finally, it will be important to understand the possible role of isocurvature fluctuations.

\section{Astrophysical Constraints on the N-flation Parameters}

The simplest realization of N-flation is that in which $N$ uncoupled, canonical fields have identical masses. As explained in [1], this model is equivalent to chaotic inflation with the potential $m^{2} \phi^{2}$. On the other hand, we have also seen that a spread in the masses will modify the scale-dependence of the spectrum. We now turn to the observational constraints that can be imposed on the parameters of N-flation. Since the spectrum depends on the 
initial field distribution, we will focus on qualitative constraints, rather than constructing detailed exclusion diagrams.

First, we can normalize the mass scale by comparing $P_{\mathcal{R}}$ to the amplitude of the temperature fluctuations observed in the CMB [43]:

$$
P_{\mathcal{R}}=\left(\frac{5}{3}\right)^{2} \frac{800 \pi^{2}}{T_{C M B}^{2}} A\left(k_{0}\right)=2.95 \times 10^{-9} A\left(k_{0}\right)
$$

where $A\left(k_{0}\right)$ parametrizes the height of the spectrum at some fiducial wavenumber $k_{0}$ and $T_{C M B}$ is expressed in micro-Kelvins. Observationally, WMAP measures $A \approx 0.78$ 44,45]. For any given initial conditions the mass $\bar{m} \equiv \sigma$ is determined by comparing (7.5) and (8.1); however, the precise result can change by a factor of a few, depending on $\alpha$ and $\beta$. Taking $\mathcal{N} \approx 60$ and initial conditions that are not correlated with the masses $(\alpha=0)$, we find

$$
\sigma \approx 1.5 \times 10^{-5} M_{P}
$$

In addition to the scalar perturbations, we must also consider the production of tensor perturbations during inflation. These are generated by quantum-mechanical fluctuations in the spacetime background itself, and have amplitude proportional to $H \propto \sqrt{V}$. One can seek these at CMB scales, through their contribution to the temperature anisotropy, through their contribution to the polarization B-mode (see e.g. [46] for the likely sensitivity of future experiments), or via a direct detection of a stochastic background of gravitational waves with a mission such as BBO [47]. Like the single-field $m^{2} \phi^{2}$ model it mimics, Nflation occurs at a comparatively high scale, and thus yields a significant tensor spectrum. However, to break the degeneracy between N-flation and single-field $m^{2} \phi^{2}$ inflation, a very accurate measurement of the tensor spectrum would be required. The exact relation between the tensor perturbations in the single-field model and in N-flation will depend on the distribution of initial vevs, as well as on $\beta$. In practice, however, the amplitudes are certainly of the same order in the two cases, though not necessarily identical. Moreover, as both N-flation and $m^{2} \phi^{2}$ produce a large tensor spectrum relative to most other inflationary models, a sufficiently tight constraint on the tensor amplitude would rule out both N-flation and single-field $m^{2} \phi^{2}$ models.

The next constraint we explore is the lower bound on $N$, arising from (6.21) and from the requirement that the individual axion vevs $\phi_{i}$ never approach $M_{P}$. Again taking $\mathcal{N} \sim 60$, we find

$$
\frac{\phi_{i}^{2}}{M_{P}^{2}} \sim \frac{240}{N}
$$


at the onset of inflation. Unless one can find reasonable string theory constructions that allow values of $N$ as high as $10^{4}$, the axion vevs in a model that produces adequate inflation will have to begin uncomfortably close to the Planck scale, though still below it. Although this situation is not entirely satisfying, it is still preferable to a single-field model in which the initial vev is parametrically large compared to the Planck scale, as it is improbable that models of the latter sort can exist in any controlled framework in string theory.

In the previous section, we showed that the spectral index is similar to that produced by single-field $m^{2} \phi^{2}$ inflation, but with somewhat more tilt at CMB scales (at least for those initial field configurations we considered explicitly.) These changes in the scalar power spectrum can be noticeable in the theoretically-favored case of the Marčenko-Pastur distribution with $\beta \sim \frac{1}{2}$, in that $n_{s}-1$ can change by around $25 \%$. Observing this difference unambiguously would require a CMB / Large Scale Structure dataset of exquisite precision, but is potentially within the reach of anticipated CMB missions.

In addition to curvature perturbations, multi-field inflationary models can also generate isocurvature (entropy) perturbations, 19 as well as non-Gaussianities [49] beyond those found in the single-field case. In this initial analysis we have ignored both of these effects, along with the possible impact of higher-order corrections to the slow roll formalism. Taking these issues in reverse order, working at lowest order in slow roll gives us a very good account of the inflationary phenomenology of the single-field $m^{2} \phi^{2}$ model. In N-flation the spread in the mass spectrum along with a generic, non-identical, set of initial vevs for the axions means that some fields will cease to be critically damped before the inflationary era comes to an end, i.e. before the second derivative of the scale factor $a(t)$ becomes non-positive. If the initial vevs are similar and $\beta$ is not very close to unity, this breakdown in slow roll will likely only occur near the end of inflation, but in the general case one needs to exercise caution when using any slow-roll results. Further, understanding the non-Gaussianities [49] and isocurvature modes [50] generated during multi-field inflation requires a calculation that extends well beyond the slow-roll approximation of the curvature perturbation, but may provide further avenues for breaking the degeneracy between N-flation and its single-field limit. We intend to address this problem directly in future work. In practice, a definitive understanding of the perturbation spectrum might require

19 See [48] for an interesting treatment of isocurvature in an assisted chaotic inflation model with $O(N)$ symmetry. This analysis corresponds to the $\beta \rightarrow 0$ limit in our terminology. With $\beta \approx 1 / 2$ the masses vary significantly and the $O(N)$ symmetry is badly broken. 
evolving the $N$ fields' perturbation equations alongside the background equations, as was done in [51] for the single-field case. This would not be prohibitively challenging: the $N$-field simulations displayed in this paper take a few seconds to complete, and adding the perturbations would roughly double the number of relevant degrees of freedom. Thus, the generic numerical problem may be computationally tractable.

Finally, it is encouraging to note that astrophysical data is rapidly reaching the point where it can put severe constraints on large classes of inflationary models. In particular, the recent analysis by Seljak et al. [52 uses a large set of astrophysical data sources to argue for tight constraints on the primordial spectrum, which rule out many "standard" models of inflation. In particular, $\lambda \phi^{4}$ is excluded at the $3 \sigma$ level, while the single-field $m^{2} \phi^{2}$ model makes predictions that differ from the central value at the $2 \sigma$ level. Obviously these constraints on the primordial spectrum will need to be confirmed (and will hopefully tighten) as further datasets become available. As noted earlier, we have not fully analyzed the inflationary predictions of N-flation in the general $\beta \neq 0$ case, and we do not compute direct astrophysical constraints on the parameter values. However, the overall size of the error ellipses in [52] suggests that observations may soon put tight constraints on the Nflation parameter space (if they do not do so already), providing further motivation for a careful analysis of the cosmological predictions of this model.

\section{Possible Exceptions to our Results}

In this section we collect the conditions and assumptions that were required in order to ensure the controllability of N-flation generally and to derive the Marčenko-Pastur spectrum.

First, in $\S 9.1$, we review the constraints implied by controllable perturbative and instanton expansions. These requirements, which were stated in [1], have little to do with

the inflationary dynamics per se; instead, they should be thought of as necessary conditions for a controllable trans-Planckian vev created by $\mathrm{N}$ string axions.

Then, in $\S 9.2$, we summarize the assumptions and constraints that apply particularly to our random matrix ensemble of N-flation models, with the intention of revealing the exceptional classes of N-flation models that might avoid our conclusions. 


\subsection{Conditions for a Trans-Planckian Displacement}

One of the main results of [1] is the construction of plausibly trans-Planckian vevs $\Delta \Phi>M_{p}$ from multiple string axions. As explained in detail in [1], radiative corrections diminish the effective size of $\Delta \Phi$ and reduce the expected scaling

$$
(\Delta \Phi)^{2} \propto N
$$

to

$$
(\Delta \Phi)^{2} \propto \frac{N}{\chi(M)}
$$

where we recall that in our notation, $P-1$ is the number of complex structure moduli, and

$$
\chi(M)=2 N-2(P-1)
$$

The result is that $\Delta \Phi / M_{p}$ can be large, but only when there are partial cancellations between $N$ and $P$. This is a powerful reason to prefer models with $\beta \sim \frac{1}{2}$, where this cancellation is almost total. (An unfortunate consequence of this numerical, rather than parametric, success is that factors such as $2 \pi$ are of considerable importance in determining the output of a given model.)

Furthermore, although one might hope to construct trans-Planckian vevs in a string vacuum with extremely weak coupling and extremely large volume, this has not yet been achieved. The problem is that the axion decay constants diminish as the volume increases, so it is not possible to work at arbitrarily large volume.

Let us therefore examine the conditions for control of the two perturbative expansions of string theory: the string loop expansion, controlled by $g_{s}$, and the sigma model expansion, controlled by $\alpha^{\prime} / R$, where $R$ is a typical length-scale in the compact space. Except when noted, we are here simply summarizing the arguments of [1].20

The leading contribution to the Planck mass from sigma model corrections is

$$
\frac{\delta_{\alpha^{\prime}} M_{p}^{2}}{M_{p}^{2}}=\frac{\chi(M) \zeta(3)}{8 \pi^{3}} \frac{\alpha^{\prime 3}}{V_{6}}
$$

which depends on $N$ through $\chi(M)$. Next, the dangerous corrections to $M_{p}$ from the string loop expansion are those that have the leading $\mathrm{N}$-scaling, namely

$$
\frac{\delta_{g_{s}} M_{p}^{2}}{M_{p}^{2}}=\sum_{q}\left(\frac{g_{s}^{2} N}{16 \pi^{2} \gamma_{q}}\right)^{q}\left(\frac{M_{k k}}{M_{p}}\right)^{2 q}
$$

20 We thank J. Maldacena for very instructive discussions of these arguments. 
In the final expression, we have included the usual field theory loop factor of $16 \pi^{2}$, as well as a parameter $\gamma_{q}$ that measures the deviation of the actual prefactor, in the string theory q-loop result, from this naive expectation. (In [1], the very conservative choice $\gamma_{q}=\frac{3}{8 \pi}$ was made. It would be worthwhile to compute this factor directly for the one-loop case.) The ratio of the Kaluza-Klein mass $M_{k k}$ to the Planck mass arises in (9.5) for the following reason. We are considering loop corrections to the four-dimensional Planck mass that arise from axions, or their moduli partners, circulating in loops. These fields have couplings of gravitational strength, and the diagrams must be cut off at the compactification scale; combining these results leads to (9.5).21

The minimal requirements for perturbative control are

$$
\begin{aligned}
& \frac{\delta_{\alpha^{\prime}} M_{p}^{2}}{M_{p}^{2}}<1 \\
& \frac{\delta_{g_{s}} M_{p}^{2}}{M_{p}^{2}}<1
\end{aligned}
$$

which imply constraints on $g_{s}$ and on the compactification volume. Among these is the important result that $(9.6)$ is most readily satisfied when $\beta \sim \frac{1}{2}$.

In addition, we must ensure that the single-instanton contribution to the superpotential is a good approximation. This follows from

$$
\rho_{i}>1 \quad i=1 \ldots N
$$

A skeptical reader may be concerned that we have dropped multi-instanton terms in the superpotential (2.5) but retained the products of two single-instanton terms in the potential. This is not inconsistent: the leading contributions to the potential from a multi-instanton term in the superpotential have the schematic forms

$$
\Delta V \propto D_{a} W_{0} e^{-2 \pi\left(\tau_{i}+\tau_{j}\right)}+\text { c.c. }
$$

where $a$ is a complex structure modulus, and

$$
\Delta V \propto W_{0} e^{-2 \pi\left(\tau_{i}+\tau_{j}\right)}+\text { c.c. }
$$

21 In [四], higher-loop terms were conservatively taken to be proportional to a single power of $\frac{M_{k k}^{2}}{M_{p}^{2}}$. Even in this case, the requirements of control can be met. 
The factor $D_{a} W_{0}$ in the former would vanish [4] if the background fluxes were imaginary self-dual. In a vacuum stabilized by nonperturbative effects, this factor will be nonvanishing, but nonperturbatively small [53]. Thus, (9.9) is negligible compared to the terms we have retained. Next, (9.10) is highly suppressed because of the smallness of $W_{0}$ : to set an appropriate overall mass scale, we needed to work with a flux configuration in which $W_{0}$ is very small [37], exactly as in [5]. Finally, notice that our random matrix arguments could be extended even to cases in which multi-instantons are relevant.

It was argued in [1] that the intersection of the constraints (9.6),(9.7),(9.8) is nonempty and includes configurations with trans-Planckian displacements. Even so, verifying this by direct computation in an explicit example would be most valuable.

\subsection{Conditions for an N-flation Model with Marčenko-Pastur Spectrum}

For definiteness, we have focused our attention on KKLT vacua in type IIB string theory. However, scenarios other than that of KKLT have some notable virtues for N-flation. Type IIA moduli stabilization with fluxes [31] produces a hierarchy between the scale of moduli stabilization and the scale at which nonperturbative effects produce axion masses. This reduces the challenge of arranging that the Kähler moduli $\rho_{i}$ are massive enough to remain at their minima during inflation. Heterotic string vacua have the advantage that the constraints of perturbative control reviewed in $\S 9.1$ can be less stringent for NS axions than for RR axions, because the relation between the compactification volume and the decay constants is different, by factors of the string coupling, in the two cases. The axions suitable for N-flation in known, stable type II vacua are RR axions whose nonperturbative potentials come from D-brane instantons, whereas in the heterotic string the relevant axions come from the NS B-field and receive their potentials from worldsheet instantons.

More importantly, for any vacuum in the above classes, the only essential property that we provided as an input to the supergravity potential (3.2) is that the axion potential arises from F-terms that vanish when the axions are at their origin. As explained in $\S 2.3$, in practice we require only the natural assumption that any F-term energy present at the axions' minimum is negligibly small compared to the energy scale of inflation. In this sense, our results are extremely general: we expect that the Marčenko-Pastur spectrum of masses is characteristic of $\mathrm{N}$-axion potentials in most of the approximately $\mathrm{F}$-flat vacua in any supergravity theory 22

22 Although our primary motivation was inflation, (4.8) is truly a result about the N-axion potential, whether or not this is relevant for inflation. 
Axion inflation around a vacuum that is not even approximately F-flat could lead to a mass matrix whose form is different from (4.7). However, we expect that in such a case the mass spectrum will be an appropriate mixture of the Wigner semicircle and MarčenkoPastur distributions. Given a specific, well-motivated model that is not F-flat, one could easily apply our methods to determine $n_{s}$.

We approximated the entries (4.2) as independent, random variables. Strong correlations among these terms would violate this assumption. However, it was shown in [54] that the Wigner law persists in ensembles with substantial correlations among the matrix entries, and it is reasonable to anticipate a similar result for the Marčenko-Pastur law. We are unaware of any motivation for a correlation that is substantial enough to invalidate (4.8). Of course, it would be extremely interesting to find exceptions; the effect of known correlations could possibly be analyzed through methods similar to those of [55]. Moreover, even in the presence of strong correlations and totally non-random behavior, some basic results are available. Using only the fact that $M(3.9)$ is positive-definite, and making no assumptions whatsoever about the statistical properties of its entries, it follows [56] that for any $i \neq j$,

$$
m_{\max }^{2}-m_{\min }^{2} \geq 2\left|M_{i j}\right|
$$

where the l.h.s., the difference between the largest and smallest eigenvalues of $M$, is known as the spread of $M$. This implies a weak but unavoidable lower bound on the difference between the largest and smallest axion masses-squared.

We did not have to assume that the distributions are Gaussian or otherwise simple, or that the mean $\mu$ is zero, because the result of Marčenko and Pastur can be shown [27] to follow without these conditions. We also did not have to take the $N \rightarrow \infty$ limit. The Marčenko-Pastur law applies as $N \rightarrow \infty$, but is an excellent approximation for the values of $N \gtrsim 200$ that were already required for $\mathrm{N}$-flation to be successful. Corrections to the moments (6.14) are of order $\frac{1}{N}$ [41] and are too small to be relevant in our results.

We did have to make a restrictive assumption (as was done in [1] for simplicity, though some statements made there do apply to general initial conditions) about the initial conditions, namely that the initial vevs obey $\phi_{i} \ll 1 \forall i$. The motion of $N$ axions with large vevs is governed by (3.10), but we have left a study of the dynamics of such a system as an interesting problem for the future.

We noted that, as a result of the renormalization of the Newton constant explored in [1] and reviewed in $\S 9.1$, models with $\beta \sim \frac{1}{2}$ are preferred. In such models the spectrum 
is typically more red, for a range of initial conditions, than in single-field $m^{2} \phi^{2}$ inflation. An obvious way to avoid this constraint would be to demonstrate that models with much smaller values of $\beta$ are in fact controllable and can give enough inflation.

\section{Conclusions}

We began with the observation that substantial cross-couplings between axions are a generic feature of $\mathrm{N}$-axion models in string theory. Moreover, complicated kinetic terms further connect the various axions. Finally, as suggested in [1], the masses of the individual fields are not identical, in general, but are spread over some range. Each of these effects can lead to nontrivial departures from the simplest realization of assisted inflation: the fields do not roll independently, and the dynamics is more complicated than that of chaotic inflation with the potential $m^{2} \phi^{2}$ [8].

When the initial axion vevs are taken to be small in units of the appropriate decay constants, the cosine potentials may be expanded, and the leading term in the resulting potential is quadratic. In this setting, one may rotate and rescale fields to produce canonical kinetic terms and a diagonal mass matrix. The result is a model specified by a spectrum of masses for $N$ uncoupled axions.

In this basis in which the axion kinetic terms are canonical and the mass matrix is diagonal, the dynamics appears relatively simple, though not quite as simple as in ordinary $m^{2} \phi^{2}$ inflation. The most massive axions fall to their minima first, diminishing the Hubble friction and releasing progressively lighter axions to fall to their minima. The Hubble parameter, and hence the spectral index of the scalar perturbations, therefore depends on the spacing in mass between adjacent axions. In any given compactification, this spacing depends on a formidable array of microscopic details. However, the statistics of the spread in masses can be characterized much more simply.

We used random matrix techniques to compute 23 the typical axion mass spectrum, taking as input the structure of the supergravity potential. We found that the spectrum is given by a simple and universal analytic formula (4.8), the Marčenko-Pastur law [39], in the limit $N \rightarrow \infty$. For realistic values of $N$, the agreement between this prediction and the results of Monte Carlo simulations of the mass spectrum is excellent.

23 The overall mass scale $\bar{m}$ must be fixed by requiring the correct amplitude of density perturbations, as in [1]. 
The shape of the Marčenko-Pastur curve (4.8) depends on a single rational parameter $\beta$ (4.11), which is determined by the dimensions of the Kähler and complex structure moduli spaces. Our results and constraints are therefore phrased in terms of $\beta$ alone. This is a rather dramatic simplification from the naive expectation that a randomly chosen model of the form (2.3) would be characterized by $\mathcal{O}(N)$ independent parameters. Even worse, in the full model (3.10) in which the initial displacements are so large that terms beyond quadratic in the axions must be kept, at least $\mathcal{O}\left(N^{2}\right)$ parameters would be required. In this sense, random matrix models of N-flation are surprisingly predictive.

We then studied the inflationary trajectory of a general N-flation model. Using the distribution (4.8) to generate an ensemble of mass matrices simulating an ensemble of Nflation models, we numerically integrated the equations of motion for this system. Next, we studied the adiabatic perturbations in a general N-flation model. We gave analytical expressions for the scalar power spectrum (7.4) and its tilt (7.16) and running (7.18) from which, given any spectrum of axion masses and choice of initial conditions, one can readily deduce the cosmological observables.

We found that the scalar power spectrum resulting from a generic N-flation model is distinguishable, in principle, from the single-field $m^{2} \phi^{2}$ inflation result. In the case of the initial conditions that we argued are most plausible, the spectral index can be written very simply in terms of $\beta$, as (7.17). For nonzero $\beta$, the spectrum is more red than in the associated single-field model; for the preferred value $\beta \approx \frac{1}{2}$ the change in $n_{s}-1$ is around $25 \%$. Let us stress here that there do exist parameter regimes, e.g. $\beta \rightarrow 0$, in which an $\mathrm{N}$-flation model is observationally similar to $m^{2} \phi^{2}$ inflation. However, to the extent that these are unusual parameter choices, we find that N-flation 'usually' differs from $m^{2} \phi^{2}$ inflation.

Because the dynamics is not independent of the initial conditions, even at late times, we presented our general results as functions of the initial conditions. For example, knowing only $\beta$ and not the initial conditions, we cannot predict the exact tilt of the scalar power spectrum, but we can make the general prediction that the spectrum will be more red than in the single-field case, by an amount that depends on $\beta$. In reasonable special cases in which the field vevs or the energy content of each field are roughly equal, the results are substantially simpler. In this connection, it would be interesting to characterize the most plausible initial conditions for models with large numbers of moduli.

Although we propose that random matrix theory is a very useful tool for analyzing Nflation, we certainly do not claim that random matrix results produce or reveal a flattening 
of the inflaton potential. These techniques merely allow us to extract a nearly-universal axion mass spectrum in a context where direct computation of the masses is unmanageable. In fact, our results imply that the scalar power spectrum of a randomly chosen N-flation model is typically more red than in $m^{2} \phi^{2}$ inflation. Thus, we actually find that a generic $\mathrm{N}$-flation potential is fractionally steeper than in the simplest model proposed in [1]. Fortunately, this slight steepening does not spoil slow-roll inflation, and, as we explained in $\S 8$, it can sometimes yield a testable prediction.

One interpretation of our results is that, in certain circumstances, assisted inflation has a novel advantage over the a priori much simpler single-field inflation models. Suppose one chooses an embedding of inflation in string theory that involves one or a few dynamical fields. The exact form of the inflaton potential will, of course, depend on the specifics of the compactification. Given an ensemble of realizations of this model, with differing choices of the microscopic parameters, the mean behavior will give only a qualitative guide to the behavior of a particular realization: fluctuations from the mean are not suppressed by large-number effects. Consider, in contrast, an ensemble of realizations of N-flation, or of some other sort of assisted inflation. The large-N simplifications of random matrix theory, as well as the well-known suppression of fluctuations from the mean, ensure that a typical realization of N-flation is very well described by the mean behavior in this ensemble of realizations. This greatly enhances the predictivity of this class of models. In a sense, this runs counter to the naive argument that the complexity of the string landscape makes it almost impossible to draw conclusions about the inflationary models that exist within it. One might seek other situations where random matrix theory can be combined with the large dimensionality of the landscape to draw generic conclusions about classes of models $36,57,58$.

In particular, it would be very interesting to find other examples in which assisted inflation becomes somewhat more predictive and less dependent on microscopic data as a consequence of large-N statistical effects. Not every assisted inflation model is usefully specified by a matrix or a set of matrices, so random matrix results per se are not always applicable. Even so, for a sufficiently large number of fields, statistical reasoning may still produce useful simplifications.

We focused, for definiteness, on KKLT compactifications of type IIB string theory, but as explained in $\S 9$, it is entirely clear that our conclusions generalize to other contexts. Even so, it would be valuable to examine the detailed properties of the N-flation potential in other string theories or in M-theory. 
We have left untouched many other interesting questions about N-flation. In particular, although we have learned something about the statistical properties of an ensemble of N-flation models, we have not provided anything like a concrete realization of the model, e.g. in a specific orientifold. This would be a worthwhile endeavor.

\section{Acknowledgements}

We thank Oliver DeWolfe, Josh Friess, Michael Haack, Simeon Hellerman, Lev Kofman, Axel Krause, Andrew Liddle, Eugene Lim, Juan Maldacena, Anupam Mazumdar, Hiranya Peiris, Douglas Stone, and Jay Wacker for valuable discussions. We are indebted to Shamit Kachru for extremely helpful correspondence. In addition, we thank Daniel Baumann for detailed comments on drafts of this paper, and Shamit Kachru, Andrew Liddle, and John McGreevy for catching an error in $\S 7$ of a preliminary draft. We thank the Aspen Center for Physics, where this project was initiated, for providing a stimulating environment. L.M. would also like to thank the Particle Theory Group at Yale, the organizers of the Dark Energy Workshop of the Arnold Sommerfeld Center, and the string theory and cosmology groups at NORDITA for their hospitality. R.E. is supported in part by the United States Department of Energy, under contract DOE-FC02-92ER-40704. L.M. is supported in part by the United States Department of Energy, under contract DE-FG02-91ER-40671.

\section{Appendix A. Statistical Suppression of Cross-Couplings}

In this Appendix we will compare the sizes of diagonal and off-diagonal elements in the mass matrix (3.9). We will show that the diagonal elements $M_{i i}$ are normally distributed around a mean that is larger, by a factor $\sim \sqrt{N+P}$, than the expected magnitude $\left\langle\left|M_{i j}\right|\right\rangle$ of off-diagonal entries $M_{i j}, i \neq j$.

As we explained in $\S 4$, the basic structure of (3.9) can be captured by

$$
M_{i j}=R_{i A} R_{j}^{A}
$$

where $R$ is a random $(N+P) \times N$ matrix that is related by a rotation to $H_{A j} \equiv D_{A} C_{j}$. The entries of $R$ may have either sign. As we demonstrated in $\S 4.1$, the entries of $R$ have a mean $\mu$ that is small compared to the standard deviation $\sigma$. In light of this property, we 
assume for simplicity 24 that the $R_{i A}$ are normally distributed around zero with variance $\sigma^{2}$.

The expected value of a diagonal element $M_{i i}$ is immediately seen to be

$$
\left\langle M_{i i}\right\rangle=(N+P) \sigma^{2}
$$

The off-diagonal elements are only slightly more involved. We start by examining

$$
M_{i j}=\sum_{A} R_{i A} R_{j A}
$$

which contains a sum of $N+P$ products of distinct elements $R_{i A} \times R_{j A}$. Using the fact that the distribution of a product of normal distributions, each with variance $\sigma^{2}$, has variance $\sigma^{4}$, and applying the central limit theorem, we find that the $M_{i j}$ are normally distributed with zero mean and variance $(N+P) \sigma^{4} .25$ Thus, we find

$$
\left\langle\left|M_{i j}\right|\right\rangle=\sqrt{\frac{2}{\pi}} \sqrt{N+P} \sigma^{2}
$$

It follows that the ratio of the expected magnitude of the diagonal terms to that of the off-diagonal terms is

$$
\left\langle\frac{M_{i i}}{\left|M_{i j}\right|}\right\rangle=\sqrt{N+P} \sqrt{\frac{\pi}{2}}
$$

which is, up to a factor very near unity, the scaling we found in our Monte Carlo simulation. Thus, given the knowledge of the form of matrix product dictated by the supergravity potential, and using our result from $\S 4.1$ that the $D_{A} C_{i}$ have mean much smaller than one, we have derived the bimodal distribution found in $\S 5$, including the $\frac{1}{\sqrt{N+P}}$ suppression of off-diagonal elements.

\section{Appendix B. Inflation in the Presence of Cross-Couplings}

In this Appendix we will study the inflationary dynamics of $\mathrm{N}$ canonically-normalized fields coupled by a general quadratic potential, in some basis in which the mass matrix is not diagonal.

24 It is straightforward to extend our arguments, though not necessarily the order-one coefficients, to much more general distributions.

25 Because we consider a sum of $N$ terms, not a mean, the scaling is superficially different from the classic application of the central limit theorem. 
Let us parametrize the mass matrix as

$$
M_{i j}=\bar{m}^{2} \delta_{i j}+\varepsilon \bar{m}^{2} \Sigma_{i j}
$$

where $\varepsilon$ is a dimensionless constant, $\bar{m}$ sets the average mass scale, and $\Sigma_{i j}$ is a symmetric matrix whose entries have mean zero and variance $\sigma^{2}$.

We would like to determine the critical value of $\varepsilon$ for which the dynamics is dominated by the diagonal terms, with no substantial corrections due to cross-couplings. Let us first compute the total potential $V$ when each of the $\phi_{i}$ is near some average value $\bar{\phi}$. We find

$$
V \sim \frac{1}{2} \bar{m}^{2} \bar{\phi}^{2}(N \pm \varepsilon N \sigma)
$$

where the factor of $N$ in the final term comes from the random walk of $\mathcal{O}\left(N^{2}\right)$ entries in $\Sigma$. (By $\pm \varepsilon N \sigma$ we are indicating the typical range of variation of $V$.)

We now observe that

$$
2 \partial_{i} V=2 m_{i}^{2} \phi_{i}+\varepsilon \bar{m}^{2} \Sigma_{i j} \phi_{j} \sim 2 m_{i}^{2} \phi_{i} \pm \varepsilon \bar{m}^{2} \sigma \sqrt{N} \bar{\phi}
$$

where we have noted that the final term is a sum of $N$ terms distributed around zero with variance $\sigma^{2}$.

With these results we can read off the slow roll parameters:

$$
\begin{gathered}
\epsilon_{i}=\frac{M_{p}^{2}}{2}\left(\frac{2 m_{i}^{2} \phi_{i} \pm \varepsilon \bar{m}^{2} \sigma \sqrt{N} \bar{\phi}}{\bar{m}^{2} \bar{\phi}^{2} N}\right)^{2}(1 \pm \varepsilon \sigma)^{-2} \\
\eta_{i i}=\frac{2 m_{i}^{2} M_{p}^{2}}{\bar{m}^{2} \bar{\phi}^{2} N}(1 \pm \varepsilon \sigma)^{-1} \\
\eta_{i j}=\frac{\varepsilon \Sigma_{i j} M_{p}^{2}}{\bar{\phi}^{2} N}(1 \pm \varepsilon \sigma)^{-1}
\end{gathered}
$$

Clearly $\varepsilon \sigma \ll 1$ suffices to arrange that $(B .5)$ is unmodified by the presence of the cross-terms $\Sigma$. Moreover, because $\left\langle\left|\Sigma_{i j}\right|\right\rangle \sim \sigma$, this same condition on $\varepsilon \sigma$ implies that $\eta_{i j}$ is negligible compared to $\eta_{i i}$. The most stringent constraint is that the cross-terms do not substantially change $\epsilon$. This requires $\varepsilon \sigma \lesssim N^{-1 / 2}$.

We conclude that $\varepsilon \sigma \lesssim N^{-1 / 2}$ is the necessary condition for the slow-roll parameters to be unmodified by the presence of quadratic cross-couplings $\Sigma_{i j}$. However, we just showed above that in the case of N-flation, $\varepsilon \sigma \sim \frac{1}{\sqrt{N+P}}$. Thus, the cross-couplings in the 
quadratic-potential limit of N-flation are naturally small enough to leave the inflationary dynamics unaffected. (We expect that cross-couplings cannot be neglected in the full $\mathrm{N}$-axion potential, as explained in §3.2.)

Two comments on this issue are in order. First, the fact that the cross-terms are dynamically unimportant does not mean that they should simply be omitted: the distribution of eigenvalues of the mass matrix is different from the distribution of diagonal elements. Second, we interpret the suppression of cross-couplings as originating in statistical considerations, not more elementary physical ones, as evidenced by the fact that the suppression factor $\varepsilon$ is the square root of an integer, and not a ratio of mass scales. 


\section{References}

[1] S. Dimopoulos, S. Kachru, J. McGreevy, and J.G. Wacker, "N-flation," hep-th/0507205.

[2] P. Binetruy and G. R. Dvali, "D-term inflation," Phys. Lett. B 388 (1996) 241, hepph/9606342;

G. R. Dvali and S. H. H. Tye, "Brane inflation," Phys. Lett. B 450, 72 (1999), hepph/9812483;

S. Alexander, "Inflation from D - Anti-D-Brane Annihilation," Phys. Rev. D 65 (2002) 023507, hep-th/0105032;

G. Dvali, Q. Shafi and S. Solganik, "D-brane Inflation," hep-th/0105203;

C.P. Burgess, M. Majumdar, D. Nolte, F. Quevedo, G. Rajesh and R.J. Zhang, "The Inflationary Brane-Antibrane Universe," JHEP 07 (2001) 047, hep-th/0105204;

J. Garcia-Bellido, R. Rabadan and F. Zamora, "Inflationary Scenarios from Branes at Angles," JHEP 0201 (2002) 036, hep-th/0112147;

K. Dasgupta, C. Herdeiro, S. Hirano and R. Kallosh, "D3/D7 Inflationary Model and M-theory," Phys. Rev. D 65 (2002) 126002, hep-th/0203019;

N. Jones, H. Stoica and S. H. H. Tye, "Brane interaction as the origin of inflation," JHEP 0207, 051 (2002), hep-th/0203163;

S. Kachru, R. Kallosh, A. Linde, J. Maldacena, L. McAllister and S. P. Trivedi, "Towards inflation in string theory," JCAP 0310, 013 (2003), hep-th/0308055;

E. Silverstein and D. Tong, "Scalar speed limits and cosmology: Acceleration from D-cceleration," Phys. Rev. D 70, 103505 (2004), hep-th/0310221;

O. DeWolfe, S. Kachru and H. L. Verlinde, "The giant inflaton," JHEP 0405, 017 (2004), hep-th/0403123;

N. Iizuka and S. P. Trivedi, "An inflationary model in string theory," Phys. Rev. D 70, 043519 (2004), hep-th/0403203;

M. Alishahiha, E. Silverstein and D. Tong, "DBI in the sky," Phys. Rev. D 70, 123505 (2004), hep-th/0404084;

J. J. Blanco-Pillado et al., "Racetrack inflation," JHEP 0411, 063 (2004), hepth/0406230;

K. Becker, M. Becker and A. Krause, "M-theory inflation from multi M5-brane dynamics," Nucl. Phys. B 715, 349 (2005), hep-th/0501130;

A. Westphal, "Eternal inflation with alpha' corrections," JCAP 0511, 003 (2005), hep-th/0507079;

J. P. Conlon and F. Quevedo, "Kaehler moduli inflation," hep-th/0509012.

[3] J. P. Hsu, R. Kallosh and S. Prokushkin, "On brane inflation with volume stabilization," JCAP 0312, 009 (2003), hep-th/0311077;

H. Firouzjahi and S. H. H. Tye, "Closer towards inflation in string theory," Phys. Lett. B 584, 147 (2004), hep-th/0312020; 
J. P. Hsu and R. Kallosh, "Volume stabilization and the origin of the inflaton shift symmetry in string theory," JHEP 0404, 042 (2004), hep-th/0402047;

S. E. Shandera, "Slow roll in brane inflation," JCAP 0504, 011 (2005), hepth/0412077.

[4] S. Giddings, S. Kachru, and J. Polchinski, "Hierarchies from Fluxes in String Compactifications," Phys. Rev. D 66 (2002) 106006, hep-th/0105097.

[5] S. Kachru, R. Kallosh, A. Linde, and S. Trivedi, "de Sitter Vacua in String Theory," Phys. Rev. D 68 (2003) 046005, hep-th/0301240.

[6] K. Dasgupta, G. Rajesh and S. Sethi, "M theory, orientifolds and G-flux," JHEP 9908, 023 (1999), hep-th/9908088;

E. Silverstein, "(A)dS backgrounds from asymmetric orientifolds," hep-th/0106209;

A. Maloney, E. Silverstein and A. Strominger, "De Sitter space in noncritical string theory," hep-th/0205316;

B. S. Acharya, "A moduli fixing mechanism in M theory," hep-th/0212294;

F. Denef, M. R. Douglas and B. Florea, "Building a better racetrack," JHEP 0406, 034 (2004), hep-th/0404257;

V. Balasubramanian, P. Berglund, J. P. Conlon and F. Quevedo, "Systematics of moduli stabilisation in Calabi-Yau flux compactifications," JHEP 0503, 007 (2005), hep-th/0502058;

F. Denef, M. R. Douglas, B. Florea, A. Grassi and S. Kachru, "Fixing all moduli in a simple F-theory compactification," hep-th/0503124;

P. S. Aspinwall and R. Kallosh, "Fixing all moduli for M-theory on K3 x K3," hepth/0506014.

[7] S. Dodelson, W. H. Kinney and E. W. Kolb, "Cosmic microwave background measurements can discriminate among inflation models," Phys. Rev. D 56, 3207 (1997), astro-ph/9702166.

[8] A. D. Linde, "Chaotic Inflation," Phys. Lett. B 129, 177 (1983).

[9] A.R. Liddle and D.H. Lyth, Cosmological Inflation and Large-Scale Structure, Cambridge University Press, Cambridge, 2000.

[10] D. H. Lyth and A. Riotto, "Particle physics models of inflation and the cosmological density perturbation," Phys. Rept. 314, 1 (1999), hep-ph/9807278.

[11] M. Kawasaki, M. Yamaguchi and T. Yanagida, "Natural chaotic inflation in supergravity," Phys. Rev. Lett. 85, 3572 (2000), hep-ph/0004243.

[12] A. Linde, "Inflation and string cosmology," eConf C040802, L024 (2004), hepth/0503195.

[13] P. Kanti and K. A. Olive, "Assisted chaotic inflation in higher dimensional theories," Phys. Lett. B 464, 192 (1999), hep-ph/9906331.

[14] N. Kaloper and L. Sorbo, "Of pNGB QuiNtessence," astro-ph/0511543. 
[15] A. R. Liddle, A. Mazumdar and F. E. Schunck, "Assisted inflation," Phys. Rev. D 58, 061301 (1998), astro-ph/9804177.

[16] P. Kanti and K. A. Olive, "On the realization of assisted inflation," Phys. Rev. D 60, 043502 (1999), hep-ph/9903524;

K. A. Malik and D. Wands, "Dynamics of assisted inflation," Phys. Rev. D 59, 123501 (1999), astro-ph/9812204.

[17] N. Kaloper and A. R. Liddle, "Dynamics and perturbations in assisted chaotic inflation," Phys. Rev. D 61, 123513 (2000), hep-ph/9910499.

[18] A. Jokinen and A. Mazumdar, "Inflation in large N limit of supersymmetric gauge theories," Phys. Lett. B 597, 222 (2004), hep-th/0406074.

[19] M. Dine and N. Seiberg, "Nonrenormalization Theorems In Superstring Theory," Phys. Rev. Lett. 57, 2625 (1986).

[20] K. Freese, J. A. Frieman and A. V. Olinto, "Natural Inflation With Pseudo - NambuGoldstone Bosons," Phys. Rev. Lett. 65, 3233 (1990).

[21] F. C. Adams, J. R. Bond, K. Freese, J. A. Frieman and A. V. Olinto, "Natural inflation: Particle physics models, power law spectra for large scale structure, and constraints from COBE," Phys. Rev. D 47, 426 (1993), hep-ph/9207245.

[22] M. Berg, M. Haack and B. Körs, "Loop Corrections to Volume Moduli and Inflation in String Theory," Phys. Rev. D 71, 026005 (2005), hep-th/0404087;

M. Berg, M. Haack and B. Körs, "On the Moduli Dependence of Nonperturbative Superpotentials in Brane Inflation," hep-th/0409282;

L. McAllister, "An inflaton mass problem in string inflation from threshold corrections to volume stabilization," hep-th/0502001.

[23] D. E. Kaplan and N. J. Weiner, "Little inflatons and gauge inflation," JCAP 0402, 005 (2004), hep-ph/0302014.

[24] M.L. Mehta, Random Matrices, Academic Press, Boston, 1990.

[25] E. Wigner, "On the Distribution of the Roots of Certain Symmetric Matrices," Ann. of Math. 67, 325 (1958).

[26] E. Wigner, "Random Matrices in Physics," SIAM Review 9, 1 (1967).

[27] Z.D. Bai, "Methodologies in Spectral Analysis of Large Dimensional Random Matrices, A Review," Statistica Sinica 9, 611 (1999).

[28] B.J.W. van Tent, "Cosmological Inflation with Multiple Fields and the Theory of Density Fluctuations," Utrecht University Ph.D. dissertation.

[29] L. Alabidi and D. H. Lyth, "Inflation models and observation," astro-ph/0510441.

[30] M. Sasaki and E. D. Stewart, "A General Analytic Formula for the Spectral Index of the Density Perturbations produced during Inflation," Prog. Theor. Phys. 95, 71 (1996), astro-ph/9507001.

[31] O. DeWolfe, A. Giryavets, S. Kachru and W. Taylor, "Type IIA moduli stabilization," JHEP 0507, 066 (2005), hep-th/0505160. 
[32] S. Gukov, C. Vafa, and E. Witten, "CFT's From Calabi-Yau Four-folds," Nucl. Phys. B 584 (2000) 69, hep-th/9906070.

[33] T. Banks, M. Dine and M. Graesser, "Supersymmetry, axions and cosmology," Phys. Rev. D 68, 075011 (2003), hep-ph/0210256.

[34] T. Banks, M. Dine, P. J. Fox and E. Gorbatov, "On the possibility of large axion decay constants," JCAP 0306, 001 (2003), hep-th/0303252.

[35] E. Witten, talk at Strings 2005;

P. Svrček and E. Witten, to appear.

[36] M. R. Douglas, "The statistics of string / M theory vacua," JHEP 0305, 046 (2003), hep-th/0303194;

S. Ashok and M. R. Douglas, "Counting flux vacua," JHEP 0401, 060 (2004), hepth/0307049;

F. Denef and M. R. Douglas, "Distributions of flux vacua," JHEP 0405, 072 (2004), hep-th/0404116;

F. Denef and M. R. Douglas, "Distributions of nonsupersymmetric flux vacua," JHEP 0503, 061 (2005), hep-th/0411183.

[37] R. Bousso and J. Polchinski, "Quantization of Four-Form Fluxes and Dynamical Neutralization of the Cosmological Constant," JHEP 0006 (2000) 006, hep-th/0004134.

[38] A.M. Tulino and S. Verdú, Random Matrix Theory and Wireless Communications, Now Publishers, Hanover, 2004.

[39] V.A. Marčenko and L.A. Pastur, "Distributions of Eigenvalues for Some Sets of Random Matrices," Math. USSR. Sbornik 1, 457 (1967).

[40] J. Wishart, "The Generalised Product Moment Distribution in Samples from a Normal Multivariate Population," Biometrika A 20, 32 (1928).

[41] I. Dumitriu and A. Edelman, "Global spectrum fluctuations for the $\beta$-Hermite and $\beta$-Laguerre ensembles via matrix models," math-ph/0510043.

[42] F. Oravecz and D. Petz, "On the eigenvalue distribution of some symmetric random matrices," Acta. Sci. Math. Szeged. 63, 383 (1997).

[43] H. V. Peiris et al., "First year Wilkinson Microwave Anisotropy Probe (WMAP) observations: Implications for inflation," Astrophys. J. Suppl. 148, 213 (2003), astroph/0302225.

[44] C. L. Bennett et al., "First Year Wilkinson Microwave Anisotropy Probe (WMAP) Observations: Preliminary Maps and Basic Results," Astrophys. J. Suppl. 148, 1 (2003), astro-ph/0302207.

[45] D. N. Spergel et al. [WMAP Collaboration], "First Year Wilkinson Microwave Anisotropy Probe (WMAP) Observations: Determination of Cosmological Parameters," Astrophys. J. Suppl. 148, 175 (2003), astro-ph/0302209. 
[46] C. Ungarelli, P. Corasaniti, R. A. Mercer and A. Vecchio, "Gravitational waves, inflation and the cosmic microwave background: Towards testing the slow-roll paradigm," Class. Quant. Grav. 22, S955 (2005), astro-ph/0504294.

[47] L. A. Boyle and P. J. Steinhardt, "Probing the early universe with inflationary gravitational waves," astro-ph/0512014.

[48] C. T. Byrnes and D. Wands, "Scale-invariant Perturbations from Chaotic Inflation," astro-ph/0512195.

[49] G. I. Rigopoulos, E. P. S. Shellard and B. W. van Tent, "Quantitative bispectra from multifield inflation," astro-ph/0511041.

[50] N. Bartolo, S. Matarrese and A. Riotto, "Adiabatic and isocurvature perturbations from inflation: Power spectra and consistency relations," Phys. Rev. D 64, 123504 (2001), astro-ph/0107502.

[51] J. A. Adams, B. Cresswell and R. Easther, "Inflationary perturbations from a potential with a step," Phys. Rev. D 64, 123514 (2001), astro-ph/0102236.

[52] U. Seljak et al., "Cosmological parameter analysis including SDSS Ly-alpha forest and galaxy bias: Constraints on the primordial spectrum of fluctuations, neutrino mass, and dark energy," Phys. Rev. D 71, 103515 (2005), astro-ph/0407372.

[53] G. Curio, A. Krause and D. Lust, "Moduli stabilization in the heterotic / IIB discretuum," hep-th/0502168.

[54] J. H. Schenker and H. Schulz-Baldes, "Semicircle law and freeness for random matrices with symmetries or correlations," MRL 12, 531 (2005), math-ph/0505003.

[55] S. H. Simon and A.L. Moustakas, "Eigenvalue Density of Correlated Complex Random Wishart Matrices," Phys. Rev. E 69, 065101 (2004), math-ph/0401038.

[56] D. S. Bernstein, Matrix Mathematics, Princeton University Press, Princeton, 2005.

[57] J. Distler and U. Varadarajan, "Random polynomials and the friendly landscape," hep-th/0507090.

[58] A. Aazami and R. Easther, "Cosmology From Random Multifield Potentials," hepth/0512050. 